\title{
Burnout among healthcare providers in the complex environment of the Middle East: a systematic review
}

\author{
Z. Chemali ${ }^{1,9^{*}}$ (D) F. L. Ezzeddine ${ }^{2}$, B. Gelaye ${ }^{2,3}$, M. L. Dossett ${ }^{4,9}$, J. Salameh ${ }^{5}$, M. Bizri ${ }^{6}$, B. Dubale ${ }^{7}$ and G. Fricchione ${ }^{8,9}$
}

\begin{abstract}
Background: Burnout is a syndrome characterized by emotional exhaustion, increased depersonalization, and a diminished sense of personal accomplishment due to chronic emotional stress at work. Burnout impacts job satisfaction, job performance, vulnerability to illnesses, and interpersonal relationships. There is a gap in the systematic data on the burden of burnout among healthcare professionals from different sectors of healthcare in Middle Eastern countries. Our objective was to examine the burden of burnout among healthcare providers in the Middle East, how it was assessed, which sectors were included, and what interventions have been used.

Methods: Articles were found through a systematic review of search results including PubMed, Web of Science (Thomson Reuters), and PsycINFO (EBSCO) using search terms reflecting burnout in Middle Eastern countries among populations of healthcare providers. Studies were included if they examined a quantitative measure of burnout among healthcare providers in the Middle East.

Results: There were 138 articles that met our inclusion criteria for this systematic review. Studies focused on burnout in the Middle East among physicians ( $N=54$ articles), nurses $(N=55)$, combined populations of healthcare workers $(N=22)$, and medical students $(N=7)$. The Maslach Burnout Inventory was the most common tool to measure burnout. Burnout is common among physicians, nurses, and other healthcare professionals, with prevalence estimates predominantly ranging between 40 and 60\%. Burnout among healthcare providers in the Middle East is associated with characteristics of their work environments, exposure to violence and terror, and emotional distress and low social support.

Conclusions: Burnout is highly prevalent among healthcare providers across countries in the Middle East. Previous studies examining burnout in this region have limitations in their methodology. More thoroughly developed epidemiologic studies of burnout are necessary. Health system strengthening is needed in a region that has endured years of ongoing conflict, and there is an urgency to design and implement programs that tackle burnout among health professionals.
\end{abstract}

Keywords: Burnout, Middle East, Health personnel

\footnotetext{
* Correspondence: zelchemali@mgh.harvard.edu

${ }^{1}$ Departments of Neurology and Psychiatry, Massachusetts General Hospital,

Boston, MA, USA

${ }^{9}$ Harvard Medical School, Boston, MA, USA

Full list of author information is available at the end of the article
}

(c) The Author(s). 2019 Open Access This article is distributed under the terms of the Creative Commons Attribution 4.0 International License (http://creativecommons.org/licenses/by/4.0/), which permits unrestricted use, distribution, and reproduction in any medium, provided you give appropriate credit to the original author(s) and the source, provide a link to the Creative Commons license, and indicate if changes were made. The Creative Commons Public Domain Dedication waiver (http://creativecommons.org/publicdomain/zero/1.0/) applies to the data made available in this article, unless otherwise stated. 


\section{Background}

Burnout is a syndrome characterized by emotional exhaustion, depersonalization, and a diminished sense of personal achievement [1]. Evidence suggests that healthcare professionals are especially susceptible to experiencing burnout [2-4], and the rise of burnout prevalence among healthcare providers in recent years has been well documented [5]. Experts in the United States have recently deemed physician burnout as a public health crisis with up to $78 \%$ of physicians at least sometimes experiencing feelings of burnout [6]. Studies have also reported high burnout rates among medical students, residents, and nurses, with the prevalence found to be up to $44.2,45$, and $50 \%$ respectively [7-9]. Rates of burnout among healthcare workers in other highincome countries have been reported to be the comparable [10-12].

Healthcare providers experiencing burnout may consequently develop symptoms such as anxiety, irritability, mood swings and depression [13-17]. Furthermore, burnout has physical health outcomes including multiple aches and pains, digestive upset, and cardiovascular risks [18-21]. Studies further demonstrate that physicians experiencing burnout are more likely to report job dissatisfaction and intention the leave the medical profession [22]. Lastly, burnout is a concern, as it not only has costly consequences for the provider, but also for the patients and the entire healthcare system. Provider wellbeing is linked to providing quality care and favorable outcomes for patients [23]. Furthermore, the impact of productivity loss related to burnout could lead to fewer healthcare resources that, in turn, can result in healthcare service waitlists and less than optimal healthcare delivery to the public. One estimate of the costs of physician cutback and early retirement suggests it totals to at least $\$ 160$ million in patient service losses [24].

While there are a number of review studies examining the prevalence and determinants of burnout in healthcare workers in developed and/or westernized countries from North America, Europe, and Australasia [25-30], there is a paucity of reviews focusing on burnout in developing and non-western countries, including Middle Eastern countries. The Middle East may be especially vulnerable to burnout due to the fragmented health systems that are in place. Many Middle Eastern countries have a critical shortage in healthcare professionals often due to brain drain. This may lead to overwork of the available workers, making them prone to burnout. In addition, as an issue specifically pertinent to the Middle East, countries like Iraq, Syria, Palestine and Yemen have been enduring years of ongoing conflict, putting an enormous strain on their healthcare systems as well as their healthcare providers.

\section{Objectives}

This study aimed to systematically review the literature on the burden of burnout among healthcare providers in the Middle East, including the construct of burnout used, which healthcare subspecialties were included, and any interventions evaluated.

This review intends to provide a foundation for future contributions aimed at reducing the burden of burnout in the Middle East.

\section{Methods}

This systematic review was conducted according to Preferred Reporting Items for Systematic.

Review and Meta-Analyses (PRISMA) guidelines (Additional file 1: Table S1) [31].

\section{Study selection and criteria for inclusion}

In PubMed, Web of Science (Thomson Reuters), and PsychINFO (EBSCO), we identified studies using search terms for burnout and Middle Eastern countries (Additional file 2: Table S2). The term "Middle East" has no clear geographic or historical definition due to complex geopolitics [32]. There is controversy on the exact countries that are classified as being in this region, though 16 countries are common in most references, which were included in this study: Bahrain, Egypt, Iran, Iraq, Israel, Jordan, Kuwait, Lebanon, Oman, Palestine, Qatar, Saudi Arabia, Syria, Turkey, United Arab Emirates, and Yemen [33-35]. All articles published prior to March 15, 2019 were eligible for inclusion. Further inclusion and selection criteria has been previously described in a companion article that examines burnout among healthcare providers in Sub-Saharan Africa [36].

\section{Data extraction and quality assessment}

Data extraction and quality assessment methods have been previously described in a companion article [36]. Study quality assessment is presented in Additional file 3: Tables S3, Additional file 4: Table S4, Additional file 5: Table S5 and Additional file 6: Table S6.

\section{Results}

The original literature search located a total of 722 articles in PubMed, 1167 articles in PsycINFO, and 645 articles in the Web of Science database (Fig. 1). Duplicate articles were removed, and 2057 remained for title review. Articles were rejected on title review for not being relevant or not meeting the search criteria. After reviewing article titles, 629 articles remained for abstract review. Candidate abstracts of the remaining 629 studies were rejected for not being relevant or not meeting the search criteria $(N=407)$. Studies in populations of healthcare providers $(N=219)$ were selected for fulltext review. In the full-text review, articles were rejected if they were qualitative studies, not available in English, did not 


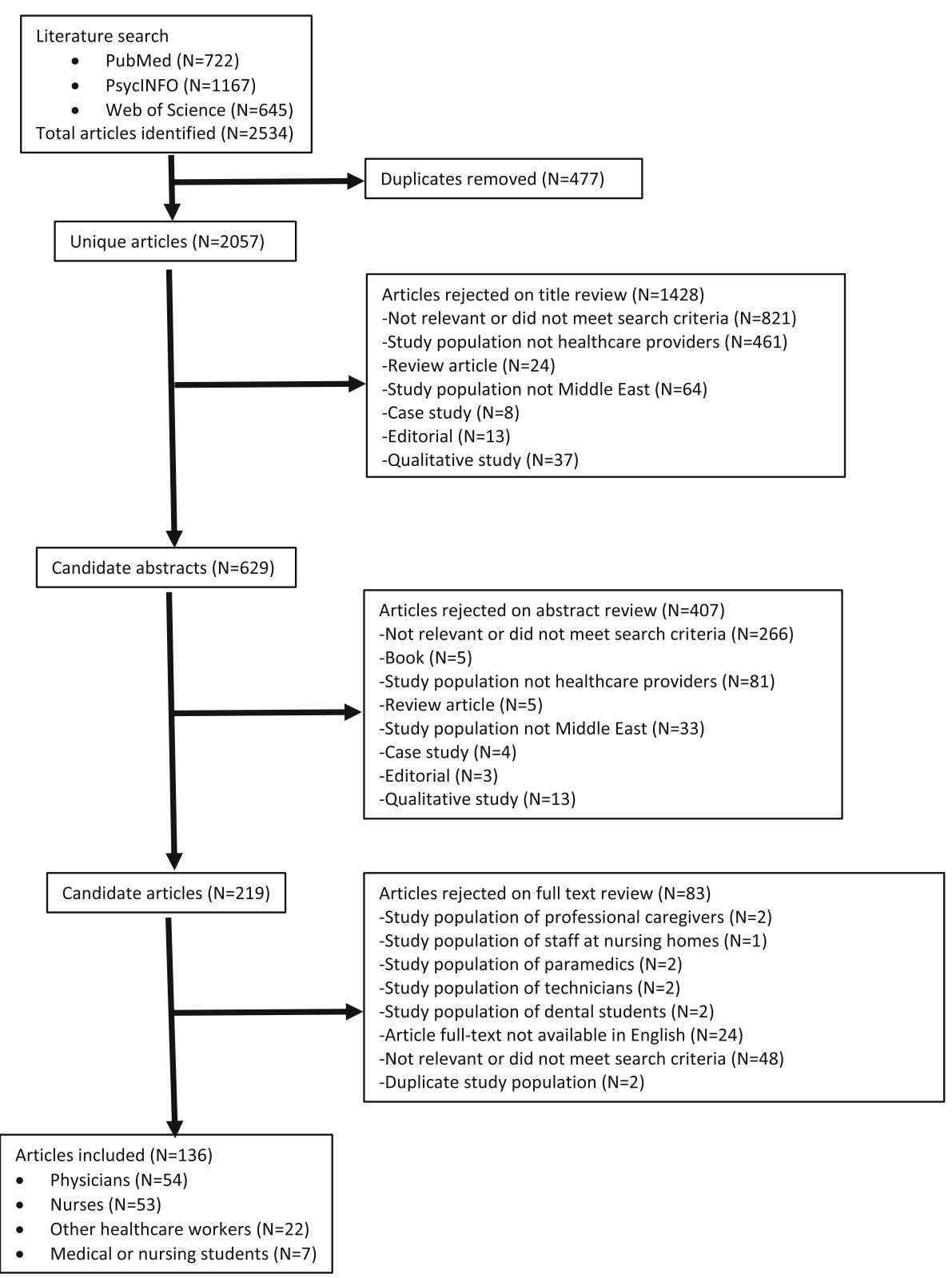

Fig. 1 Flowchart of systematic literature review

include healthcare providers, or were not relevant to the search criteria $(N=83)$.

A total of 136 articles met our inclusion criteria for this systematic review. Articles examined burnout in the Middle East among physicians $(N=54)$, nurses $(N=53)$, combined populations of healthcare workers $(N=22)$, and medical students $(N=7)$. Six articles examined burnout among healthcare professionals in Egypt, 46 in Turkey, 18 in Saudi Arabia, 29 in Iran, and 19 in Israel, 1 in United Arab Emirates, 3 in Qatar, 1 in Yemen, 8 in Lebanon, 2 in Palestine, 1 in Bahrain, and 2 in Jordan. Of the 138 eligible articles for inclusion, 130 used versions of the Maslach Burnout Inventory to measure burnout. One study in
Saudi Arabia examined burnout among National Guard Physicians using the Oldenburg Burnout Inventory. One Israeli study measured burnout among physicians using the burnout subscale of the Professional Quality of Life Scale (ProQOL). Additionally, three Israeli studies measured burnout using the Shirom-Melamed Burnout Questionnaire among physicians ( $\mathrm{N}=2$ studies) and nurses $(N=1)$. Lastly, two studies used the Burnout Measure to measure burnout among medical students in Lebanon.

\section{Burnout among physicians}

Fifty-four articles examined burnout among physicians in Middle Eastern countries, comprising a total of 8588 
participants across Egypt, Bahrain, Iran, Israel, Lebanon, Oman, Saudi Arabia, Turkey, Qatar, United Arab Emirates, and Yemen (Table 1). In 49 of the studies, burnout was assessed using the MBI [37-80] or an abbreviated MBI [81-83]. One study used the Oldenburg Burnout Inventory [84], one study used the burnout scale of the ProQOL [85], one study used the Burnout Measure [86], and two studies used the Shirom-Melamed Burnout Questionnaire [87, 88]. One study in Israel used a single-item measure adapted from the full MBI ("How often do you feel burned out from your work?") to assess burnout on a 7 point Likert scale [89]. Another study in Israel also used a single measure item adapted from the full MBI ("I have become more indifferent towards patients since I started this work") [90].

High levels of burnout were reported among physicians across the Middle Eastern countries. Among physicians undergoing residency training in the United Arab Emirates $(N=302), 70 \%$ of the participants reported overall burnout, $75.5 \%$ reported moderate-to-high emotional exhaustion, $84 \%$ had high depersonalization, and $74 \%$ experienced a diminished sense of personal accomplishment [37]. Among doctors working at a tertiary care hospital in Saudi Arabia $(N=96)$, the prevalence of reported high emotional exhaustion, high depersonalization and reduced personal exhaustion were 68.8, 63.6, and 38.5\% respectively [39]. A study in Egypt found that more than half of anesthesiologists with academic careers $(N=98)$ met the criteria for all burnout subscales [74]. Among Iranian female emergency medicine physicians $(N=85)$, the level of burnout in the three subscales of emotional exhaustion, depersonalization, and perceived low accomplishment was high in 84.5 , 48.1, and $80.5 \%$ of participants respectively [76]. A study in Yemen reported that among doctors $(N=$ $356), 63.2 \%$ were identified as experiencing a high degree of burnout in at least one category of burnout, and those working more than $40 \mathrm{~h}$ a week had a 2.8 times higher risk of reporting high levels of burnout compared to those working less than $40 \mathrm{~h}$ a week [42]. Among primary care physicians $(N=144)$ in Qatar, the overall prevalence of burnout according to the MBI was $16.1 \%$ [72]. A study assessing burnout among Israeli burn specialist clinicians $(N=55), 38.2 \%$ of them reported high levels of burnout on all three subscales of the MBI [59]. One third of internal medicine residents met the criteria for clinically significant burnout according to an assessment done in Turkey $(N=33)$ [41]. A study conducted in Lebanon found that among physicians undergoing residency training $(N=155), 80 \%$ of the sample had reported a high level of burnout in at least one domain [52]. Among secondary doctors in Bahrain $(N=230)$, the reported prevalence of high emotional exhaustion, high depersonalization, and diminished sense of personal accomplishment were 43.1, 70.6 , and $11.1 \%$ respectively [61].

\section{Burnout among nurses}

We found 53 studies that assessed burnout among a total of 10,992 nurses in Palestine, Iran, Turkey, Saudi Arabia, Egypt, Israel, and Jordan (Table 2). All of the 53 studies used the MBI to measure burnout [91-143].

Nurses reported high levels of burnout (Table 2). For example, among nurses from five private hospitals in Palestine $(N=152)$, burnout measures were high on the emotional exhaustion (mean \pm standard deviation (SD): $23.48 \pm 10.23)$, depersonalization $(5.42 \pm 5.61)$, and personal achievement $(34.14 \pm 9.44)$ subscales of the MBI [91]. A study conducted among Iranian nurses working at public hospitals $(N=180)$ found that $25 \%$ of the participants met the criteria for burnout according to the MBI [134]. A study of nurses in Israel $(N=39)$ reported that $30.8 \%$ of participants reported emotional exhaustion very frequently, $5.1 \%$ have a cynical feeling towards their job often, and $15.4 \%$ rarely experience professional selfactualization [109]. Among mental health nurses in Jordan $(N=181), 55 \%$ of them reported high level of burnout in the subscale of emotional exhaustion, 50\% reported high depersonalization, and 50\% reported a reduced sense of personal accomplishment [113].

\section{Burnout among combined populations of healthcare workers}

There were 22 articles that studied burnout among combined populations of healthcare workers (Table 3). Ten studies were based in Turkey, six studies in Iran, three studies in Lebanon, one each in Egypt, Israel, and Palestine. All of the 22 studies used the MBI to assess burnout [144-165]. High levels of burnout were reported in these populations. For example, among employees working at an emergency department in Lebanon $(N=$ 256), the prevalence of reported high emotional exhaustion, depersonalization, and reduced sense of personal accomplishment was 54.9, 43.5, and $44.5 \%$ respectively [148]. A study in Iran on mental health service providers $(N=100)$, burnout scores were high in emotional exhaustion ((mean $\pm \mathrm{SD})$ : $24.9 \pm 6.9)$, depersonalization (9.3 \pm 2.1 ), and diminished sense of personal accomplishment $(35.5 \pm 7.0)$ [150]. Among oncology employees in Turkey $(N=90)$, reported prevalence of emotional exhaustion, depersonalization, and reduced sense of personal achievement was 42,20 , and $35.6 \%$ respectively [153].

\section{Burnout among medical students}

A total of seven articles examined burnout among medical students in Oman, Saudi Arabia, Iran, Turkey, and Lebanon (Table 4). Among medical students in Oman $(N=662)$, the prevalence of overall severe burnout was $7.4 \%$ as assessed by the MBI [166]. Two studies in Saudi Arabia used the MBI and reported that among 249 and 276 medical students, the overall burnout prevalence 


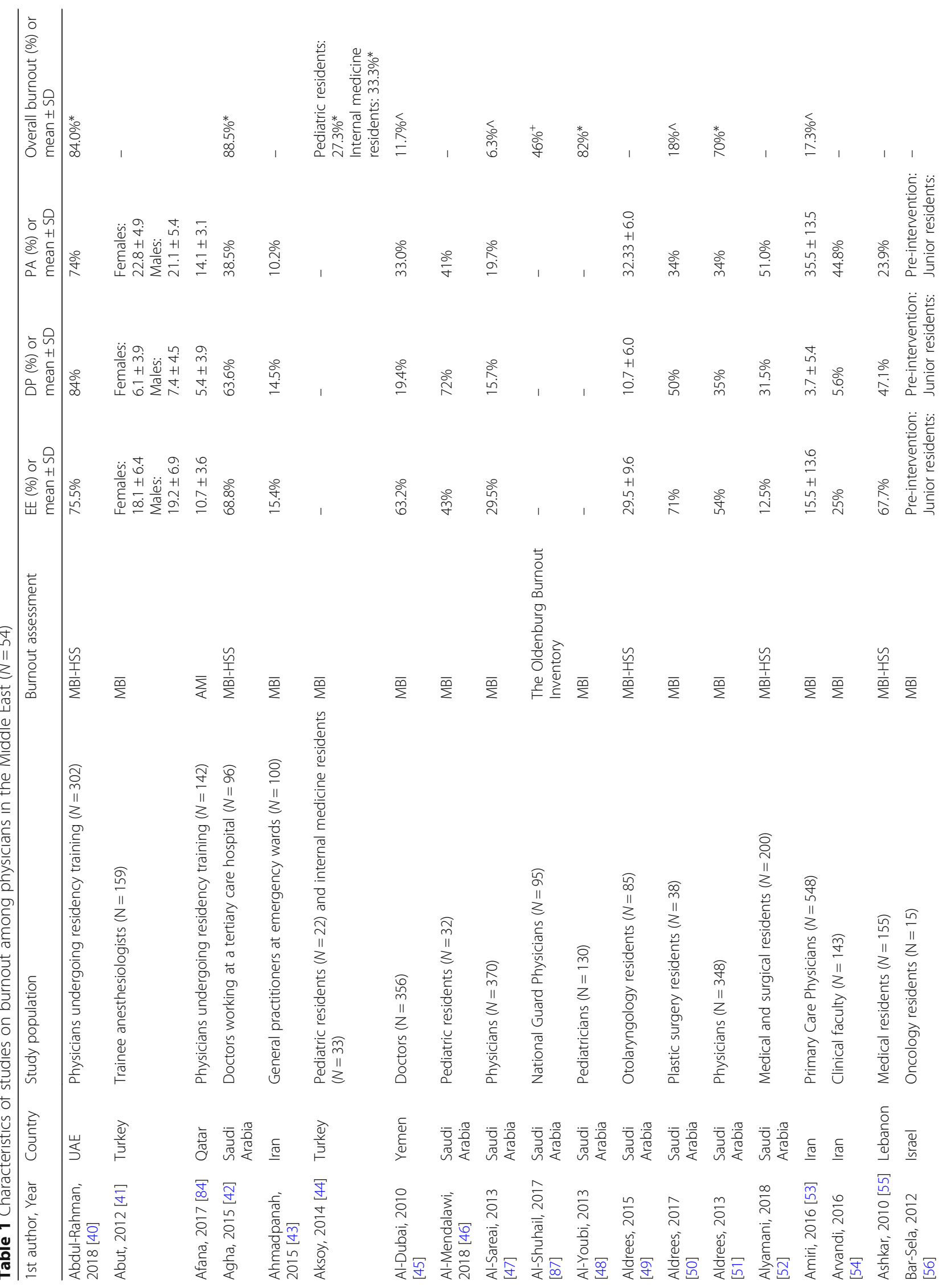




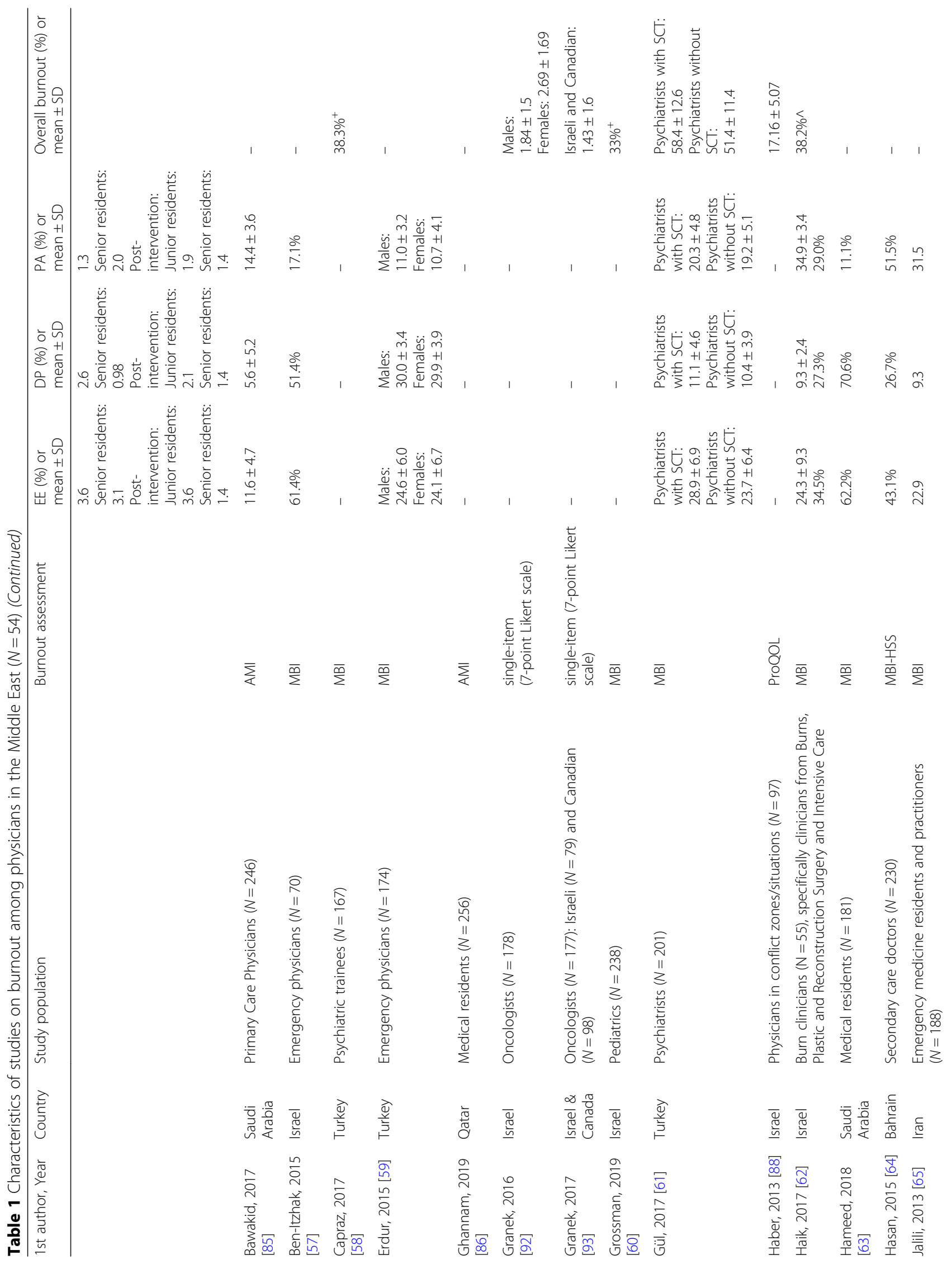




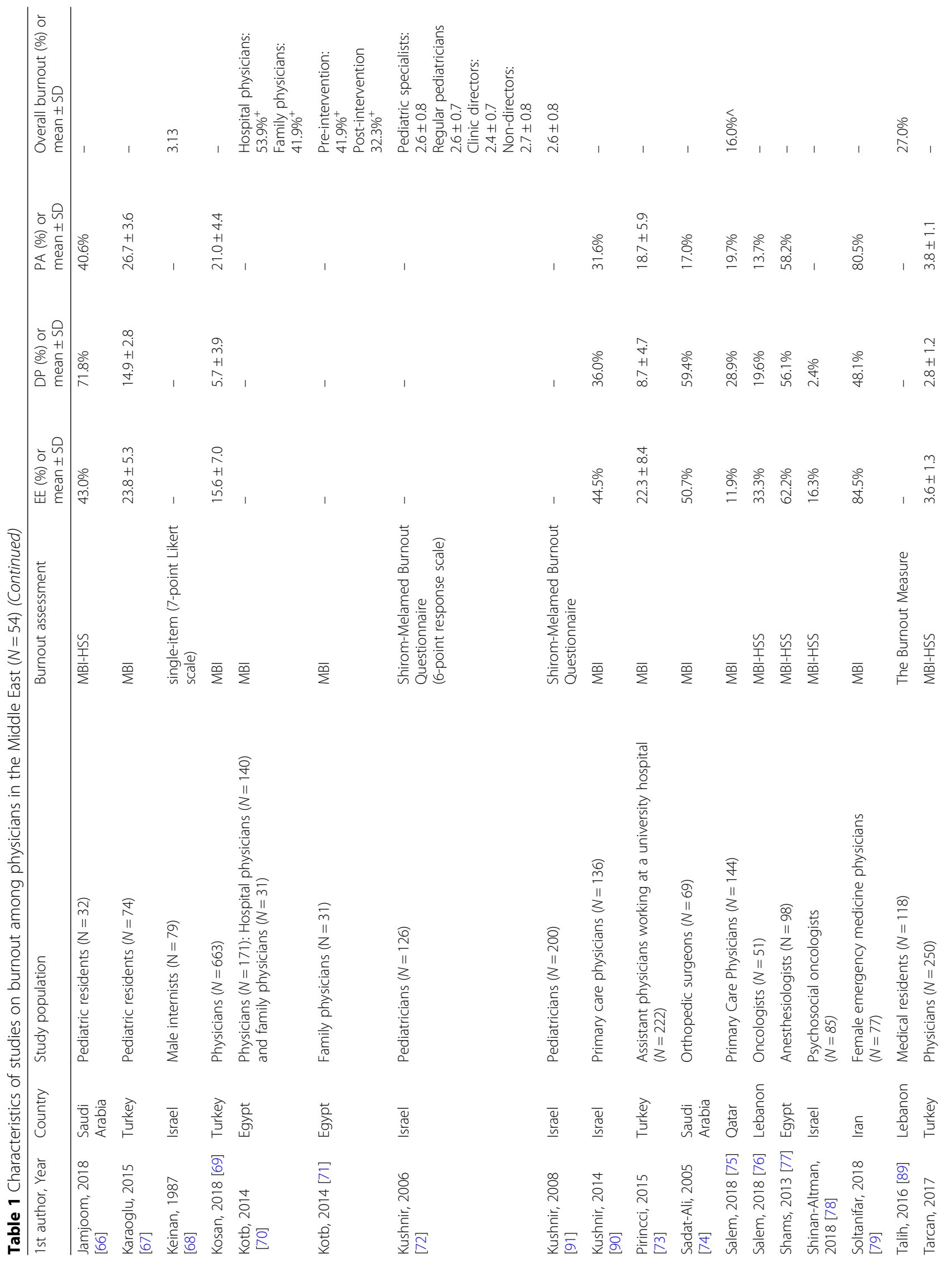




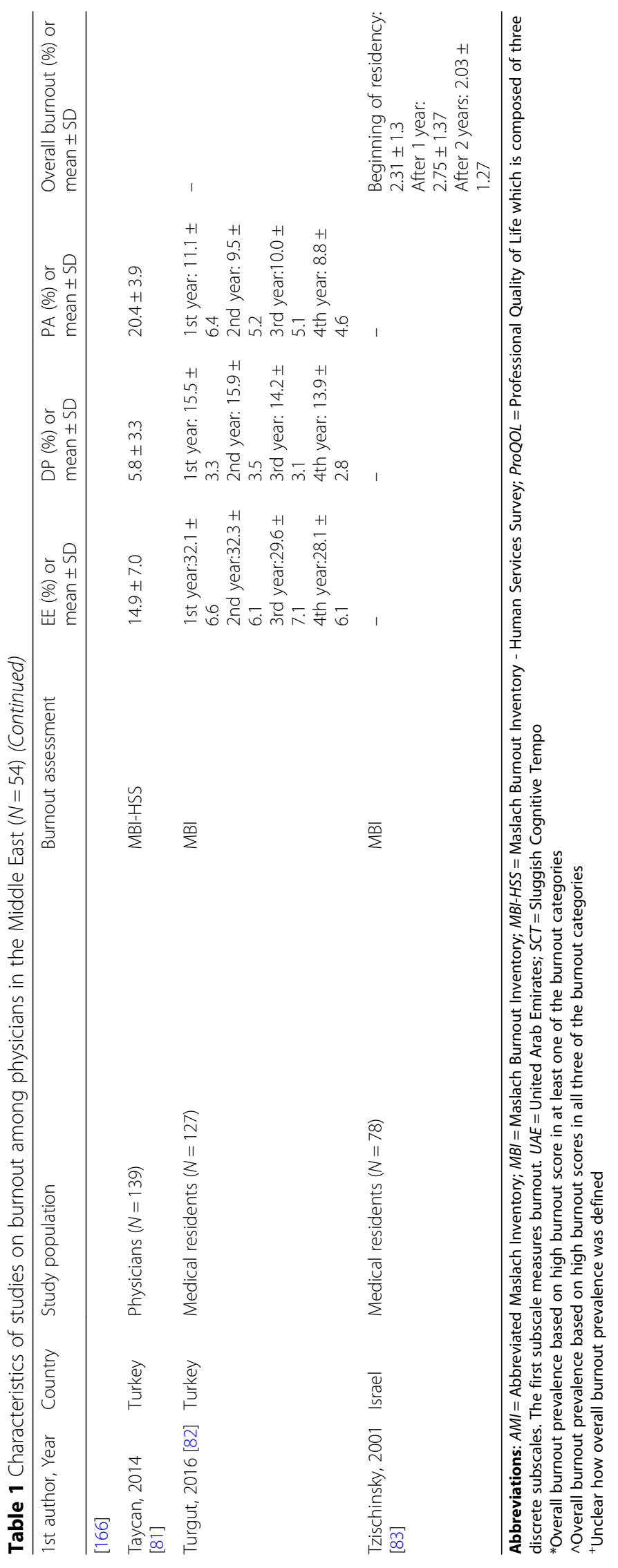


Table 2 Characteristics of studies on burnout among nurses in Middle East $(N=53)$

\begin{tabular}{|c|c|c|c|c|c|c|c|}
\hline $\begin{array}{l}\text { 1st author, } \\
\text { Year }\end{array}$ & Country & Study population & $\begin{array}{l}\text { Burnout } \\
\text { assessment }\end{array}$ & $\begin{array}{l}\mathrm{EE}(\%) \text { or } \\
\text { mean } \pm \mathrm{SD}\end{array}$ & $\begin{array}{l}\mathrm{DP}(\%) \text { or } \\
\text { mean } \pm \mathrm{SD}\end{array}$ & $\begin{array}{l}\mathrm{PA}(\%) \text { or } \\
\text { mean } \pm \mathrm{SD}\end{array}$ & $\begin{array}{l}\text { Overall } \\
\text { Burnout (\%) } \\
\text { or mean } \pm \\
\text { SD }\end{array}$ \\
\hline $\begin{array}{l}\text { Abushaikha, } \\
2009[94] g\end{array}$ & Palestine & Nurses from 5 private hospitals $(N=152)$ & $\mathrm{MBI}$ & $23.5 \pm 10.2$ & $5.4 \pm 5.6$ & $34.1 \pm 9.4$ & - \\
\hline $\begin{array}{l}\text { Ahmadi, } 2014 \\
\text { [95] }\end{array}$ & Iran & $\begin{array}{l}\text { Nurses from a university affiliated hospital } \\
(N=100) \text { : emergency }(N=28) \text {, orthopedic } \\
(N=22) \text {, dialysis wards }(N=19) \text {, and ICU } \\
(N=31)\end{array}$ & $\mathrm{MBI}$ & - & - & - & $\begin{array}{l}\text { Emergency: } \\
\text { 17.0\% } \\
\text { Orthopedic: } \\
18.6 \%^{+} \\
\text {Dialysis: } \\
29.0 \% \%^{+} \\
\text {ICU: } \\
8.7 \%^{+}\end{array}$ \\
\hline $\begin{array}{l}\text { Akkus, } 2010 \\
{[96]}\end{array}$ & Turkey & $\begin{array}{l}\text { Nurses from stem cell transplantation units } \\
(N=57)\end{array}$ & $\mathrm{MBI}$ & $15.1 \pm 7.5$ & $3.7 \pm 3.3$ & $12.2 \pm 5.1$ & - \\
\hline $\begin{array}{l}\text { Akman, } 2016 \\
{[97]}\end{array}$ & Turkey & Pediatric nurses $(N=165)$ & $\mathrm{MBI}$ & $\begin{array}{l}\text { high job } \\
\text { satisfaction } \\
\text { scores: } \\
19.1 \pm 6.2 \\
\text { low job } \\
\text { satisfaction } \\
\text { scores: } \\
26.9 \pm 6.2\end{array}$ & $\begin{array}{l}\text { high job } \\
\text { satisfaction } \\
\text { scores: } \\
5.9 \pm 3.9 \\
\text { low job } \\
\text { satisfaction } \\
\text { scores: } 9.2 \pm \\
4.9\end{array}$ & $\begin{array}{l}\text { high job } \\
\text { satisfaction } \\
\text { scores: } \\
11.9 \pm 5.8 \\
\text { low job } \\
\text { satisfaction } \\
\text { scores: } 9.8 \pm \\
5.1\end{array}$ & - \\
\hline $\begin{array}{l}\text { Al-Turki, } 2010 \\
{[98]}\end{array}$ & $\begin{array}{l}\text { Saudi } \\
\text { Arabia }\end{array}$ & $\begin{array}{l}\text { Multi-national nurses from a tertiary care } \\
\text { hospital }(N=189)\end{array}$ & $\mathrm{MBI}$ & $45.0 \%$ & $42.0 \%$ & $29.0 \%$ & - \\
\hline $\begin{array}{l}\text { Al-Turki, } 2010 \\
\text { [99] }\end{array}$ & $\begin{array}{l}\text { Saudi } \\
\text { Arabia }\end{array}$ & $\begin{array}{l}\text { Nurses from a tertiary care hospital } \\
(N=37)\end{array}$ & $\mathrm{MBI}$ & $46.0 \%$ & $49.0 \%$ & $17.0 \%$ & - \\
\hline $\begin{array}{l}\text { Alharbi, } 2016 \\
{[100]}\end{array}$ & $\begin{array}{l}\text { Saudi } \\
\text { Arabia }\end{array}$ & Critical care nurses $(N=150)$ & $\mathrm{MBI}$ & $35.2 \pm 8.9$ & $16.3 \pm 5.2$ & $33.9 \pm 8.0$ & - \\
\hline $\begin{array}{l}\text { Alimoglu, } 2005 \\
\text { [101] }\end{array}$ & Turkey & Nurses $(N=141)$ & $\mathrm{MBI}$ & $19.2 \pm 6.9$ & $5.2 \pm 3.6$ & $20.8 \pm 4.0$ & - \\
\hline $\begin{array}{l}\text { Altun, } 2002 \\
{[102]}\end{array}$ & Turkey & Nurses $(N=160)$ & $\mathrm{MBI}$ & $17.3 \pm 7.7$ & $4.6 \pm 4.3$ & $22.8 \pm 5.9$ & - \\
\hline $\begin{array}{l}\text { Anwar, } 2017 \\
{[103]}\end{array}$ & Egypt & Nurses from a university hospital $(N=227)$ & MBI-HSS & $\begin{array}{l}24.6 \pm 6.8 \\
27 \%\end{array}$ & $\begin{array}{l}12.1 \pm 4.1 \\
48 \%\end{array}$ & $\begin{array}{l}28.4 \pm 6.3 \\
78 \%\end{array}$ & - \\
\hline $\begin{array}{l}\text { Arslan, } 2016 \\
{[104]}\end{array}$ & Turkey & Nurses from 16 hospitals $(N=799)$ & $\mathrm{MBI}$ & $16.4 \pm 7.1$ & $5.4 \pm 4.2$ & $10.1 \pm 5.8$ & - \\
\hline $\begin{array}{l}\text { Azmoon, } 2018 \\
{[105]}\end{array}$ & Iran & Nurses from teaching hospitals $(N=522)$ & $\mathrm{MBI}$ & $13.1 \pm 6.6$ & $28.5 \pm 9.1$ & $18.4 \pm 7.1$ & - \\
\hline $\begin{array}{l}\text { Bagheri, } 2019 \\
{[106]}\end{array}$ & Iran & Nurses from 4 teaching hospitals $(N=684)$ & $\mathrm{MBI}$ & $38.7 \%$ & $30.4 \%$ & $24.6 \%$ & - \\
\hline $\begin{array}{l}\text { Bakir, } 2010 \\
{[107]}\end{array}$ & Turkey & Military nurses $(N=377)$ & $\mathrm{MBI}$ & $\begin{array}{l}\text { Age (year) } \\
\leq 29: \\
24.8 \pm 6.9 \\
\text { Age (year) } \\
\geq 30 \text { : } \\
12.1 \pm 10.7\end{array}$ & $\begin{array}{l}\text { Age (year) } \\
\leq 29: \\
10.7 \pm 3.7 \\
\text { Age (year) } \\
\geq 30: \\
25.3 \pm 7.3\end{array}$ & $\begin{array}{l}\text { Age (year) } \\
\leq 29: \\
20.8 \pm 5.1 \\
\text { Age (year) } \\
\geq 30: \\
20.7 \pm 5.5\end{array}$ & - \\
\hline $\begin{array}{l}\text { Chayu, } 2011 \\
\text { [108] }\end{array}$ & Israel & Nephrology nurses $(N=132)$ & $\begin{array}{l}\text { MBI and Shirom- } \\
\text { Melamed Burnout } \\
\text { Questionnaire } \\
\text { (SMBD) }\end{array}$ & $3.2 \pm 1.3$ & $1.8 \pm 0.8$ & $2.1 \pm 0.9$ & \\
\hline $\begin{array}{l}\text { Darban, } 2016 \\
{[109]}\end{array}$ & Iran & Nurses $(N=60)$ & $\mathrm{MBI}$ & - & - & - & $\begin{array}{l}\text { Pre- } \\
\text { intervention: } \\
60.8 \pm 7.8 \\
\text { After: } 60.9 \pm \\
7.9 \\
\text { After } 1 \\
\text { month: } \\
61.0 \pm 8.0 \\
\text { Study group }\end{array}$ \\
\hline
\end{tabular}


Table 2 Characteristics of studies on burnout among nurses in Middle East ( $N=53$ ) (Continued)

\begin{tabular}{|c|c|c|c|c|c|c|c|}
\hline $\begin{array}{l}\text { 1st author, } \\
\text { Year }\end{array}$ & Country & Study population & $\begin{array}{l}\text { Burnout } \\
\text { assessment }\end{array}$ & $\begin{array}{l}\mathrm{EE}(\%) \text { or } \\
\text { mean } \pm \mathrm{SD}\end{array}$ & $\begin{array}{l}\mathrm{DP}(\%) \text { or } \\
\text { mean } \pm \mathrm{SD}\end{array}$ & $\begin{array}{l}\mathrm{PA}(\%) \text { or } \\
\text { mean } \pm \mathrm{SD}\end{array}$ & $\begin{array}{l}\text { Overall } \\
\text { Burnout (\%) } \\
\text { or mean } \pm \\
\text { SD }\end{array}$ \\
\hline & & & & & & & $\begin{array}{l}\text { Pre- } \\
\text { intervention: } \\
61.1 \pm 8.0 \\
\text { After: } 58.8 \pm \\
7.6 \\
\text { After } 1 \\
\text { month: } \\
54.6 \pm 7.0\end{array}$ \\
\hline $\begin{array}{l}\text { Demir, } 2003 \\
\text { [110] }\end{array}$ & Turkey & $\begin{array}{l}\text { Nurses at university and state hospitals } \\
(N=333)\end{array}$ & $\mathrm{MBI}$ & $\begin{array}{l}\text { state } \\
\text { hospitals: } \\
19.1 \pm 5.7 \\
\text { university } \\
\text { hospitals: } \\
17.2 \pm 6.0\end{array}$ & $\begin{array}{l}\text { state } \\
\text { hospitals: } \\
5.6 \pm 3.6 \\
\text { university } \\
\text { hospitals: } \\
4.7 \pm 3.1\end{array}$ & $\begin{array}{l}\text { state } \\
\text { hospitals: } \\
20.3 \pm 4.1 \\
\text { university } \\
\text { hospitals: } \\
20.6 \pm 4.1\end{array}$ & - \\
\hline $\begin{array}{l}\text { Dor, } 2018 \\
{[111]}\end{array}$ & Israel & $\begin{array}{l}\text { Hospital nurses }(N=278) \text { and community } \\
\text { nurses }(N=179)\end{array}$ & $\mathrm{MBI}$ & $\begin{array}{l}\text { Hospital: } \\
3.0 \pm 1.0 \\
\text { Community: } \\
2.4 \pm 0.9\end{array}$ & $\begin{array}{l}\text { Hospital: } \\
2.2 \pm 0.9 \\
\text { Community: } \\
2.0 \pm 0.9\end{array}$ & $\begin{array}{l}\text { Hospital: } \\
2.1 \pm 0.6 \\
\text { Community: } \\
2.0 \pm 2.7\end{array}$ & - \\
\hline $\begin{array}{l}\text { Emold, } 2011 \\
\text { [112] }\end{array}$ & Israel & Nurses from 6oncology units $(N=39)$ & $\mathrm{MBI}$ & $30.8 \%$ & $5.1 \%$ & $15 \%$ & - \\
\hline $\begin{array}{l}\text { Farahbod, } \\
2015[113]\end{array}$ & Iran & $\begin{array}{l}\text { Nurses at a trauma referral teaching } \\
\text { hospital }(N=214)\end{array}$ & MBI & $24.7 \pm 12.2$ & $4.7 \pm 5.1$ & $41.1 \pm 9.6$ & - \\
\hline $\begin{array}{l}\text { Gholami, } 2016 \\
\text { [114] }\end{array}$ & Iran & Nurses $(N=415)$ & MBI & $25.1 \pm 12.4$ & $5.9 \pm 5.1$ & $33.3 \pm 9.6$ & - \\
\hline $\begin{array}{l}\text { Gunusen, } 2010 \\
{[115]}\end{array}$ & Turkey & Nurses $(N=108)$ & MBI & $\begin{array}{l}\text { Pre- } \\
\text { intervention: } \\
\text { Coping } \\
\text { group: } \\
20.9 \pm 4.7 \\
\text { Control } \\
\text { group: } \\
21.4 \pm 4.2 \\
\text { Post- } \\
\text { intervention: } \\
\text { Coping } \\
\text { group: } \\
\text { 20.9 } \pm 4.7 \\
\text { Control } \\
\text { group: } \\
21.4 \pm 4.2\end{array}$ & $\begin{array}{l}\text { Pre- } \\
\text { intervention: } \\
\text { Coping } \\
\text { group: } \\
5.8 \pm 3.6 \\
\text { Control } \\
\text { group: } \\
5.0 \pm 3.1 \\
\text { Post- } \\
\text { intervention: } \\
\text { Coping } \\
\text { group: } \\
\text { 4.6 } \pm 3.2 \\
\text { Control } \\
\text { group: } 5.4 \pm \\
2.7\end{array}$ & $\begin{array}{l}\text { Pre- } \\
\text { intervention: } \\
\text { Coping } \\
\text { group: } \\
19.7 \pm 3.4 \\
\text { Control } \\
\text { group: } \\
19.7 \pm 3.6 \\
\text { Post- } \\
\text { intervention: } \\
\text { Coping } \\
\text { group: } \\
\text { 21.4 } \pm 3.5 \\
\text { Control } \\
\text { group: } \\
\text { 19.9 } \pm 3.1\end{array}$ & - \\
\hline $\begin{array}{l}\text { Hamaideh, } \\
2011[116]\end{array}$ & Jordan & Mental health nurses $(N=181)$ & $\mathrm{MBI}$ & $\begin{array}{l}24.0 \pm 13.9 \\
55 \%\end{array}$ & $\begin{array}{l}7.0 \pm 7.1 \\
50 \%\end{array}$ & $\begin{array}{l}31.6 \pm 11.5 \\
50 \%\end{array}$ & - \\
\hline $\begin{array}{l}\text { lecovich, } 2017 \\
\text { [117] }\end{array}$ & Israel & Nurses in long-term care facilities $(N=154)$ & $\mathrm{MBI}$ & $25.6 \pm 10.5$ & $10.2 \pm 5.7$ & $20.1 \pm 8.1$ & - \\
\hline $\begin{array}{l}\text { Ilhan, } 2008 \\
{[118]}\end{array}$ & Turkey & Nurses $(N=418)$ & $\mathrm{MBI}$ & $18.0 \pm 6.3$ & $5.7 \pm 3.9$ & $19.8 \pm 4.7$ & - \\
\hline $\begin{array}{l}\text { Kapucu, } 2009 \\
{[119]}\end{array}$ & Turkey & Nurses from hemodialysis units $(N=95)$ & $\mathrm{MBI}$ & $16.0 \pm 6.3$ & $4.7 \pm 3.2$ & $21.0 \pm 4.6$ & - \\
\hline $\begin{array}{l}\text { Karadag, } 2017 \\
{[120]}\end{array}$ & Turkey & Nurses from a state hospital $(N=118)$ & $\mathrm{MBI}$ & $48.9 \pm 6.1$ & $5.8 \pm 3.5$ & $11.2 \pm 4.6$ & - \\
\hline $\begin{array}{l}\text { Karakoc, } 2016 \\
{[121]}\end{array}$ & Turkey & $\begin{array}{l}\text { Nurses working in dialysis centers } \\
(N=171)\end{array}$ & MBI & $14.0 \pm 7.3$ & $4.4 \pm 3.4$ & $20.8 \pm 4.1$ & - \\
\hline $\begin{array}{l}\text { Karaman, } 2017 \\
{[122]}\end{array}$ & $\begin{array}{l}\text { Turkey } \\
\text { and Iran }\end{array}$ & $\begin{array}{l}\text { Nurses working in surgical clinics }(N=179) \\
\text { in Turkey }(N=87) \text { and Iran }(N=97)\end{array}$ & $\mathrm{MBI}$ & $\begin{array}{l}\text { Turkey: } \\
28.3 \pm 6.9 \\
\text { Iran: } \\
25.5 \pm 9.2\end{array}$ & $\begin{array}{l}\text { Turkey: } \\
10.2 \pm 3.4 \\
\text { Iran: } 9.4 \pm 3.9\end{array}$ & $\begin{array}{l}\text { Turkey: } \\
29.5 \pm 5.0 \\
\text { Iran: } 31.6 \pm \\
5.2\end{array}$ & - \\
\hline Kavurmacl, & Turkey & Hemodialysis nurses $(N=32)$ & $\mathrm{MBI}$ & $17.1 \pm 8.3$ & $5.9 \pm 4.1$ & $20.6 \pm 4.1$ & - \\
\hline
\end{tabular}


Table 2 Characteristics of studies on burnout among nurses in Middle East ( $N=53$ ) (Continued)

\begin{tabular}{|c|c|c|c|c|c|c|c|}
\hline $\begin{array}{l}\text { 1st author, } \\
\text { Year }\end{array}$ & Country & Study population & $\begin{array}{l}\text { Burnout } \\
\text { assessment }\end{array}$ & $\begin{array}{l}\mathrm{EE}(\%) \text { or } \\
\text { mean } \pm \mathrm{SD}\end{array}$ & $\begin{array}{l}\mathrm{DP}(\%) \text { or } \\
\text { mean } \pm \mathrm{SD}\end{array}$ & $\begin{array}{l}\mathrm{PA}(\%) \text { or } \\
\text { mean } \pm \mathrm{SD}\end{array}$ & $\begin{array}{l}\text { Overall } \\
\text { Burnout (\%) } \\
\text { or mean } \pm \\
\text { SD }\end{array}$ \\
\hline \multicolumn{8}{|l|}{2014 [123] } \\
\hline $\begin{array}{l}\text { Kutluturkan, } \\
2016[124]\end{array}$ & Turkey & Oncology nurses $(N=140)$ & $\mathrm{MBI}$ & $\begin{array}{l}\text { (median): } \\
24.0\end{array}$ & $\begin{array}{l}\text { (median): } \\
9.0\end{array}$ & $\begin{array}{l}\text { (median): } \\
16.0\end{array}$ & - \\
\hline $\begin{array}{l}\text { KızılCI, } 2012 \\
{[125]}\end{array}$ & Turkey & $\begin{array}{l}\text { Nurses working in academic institutions } \\
(N=94)\end{array}$ & $\mathrm{MBI}$ & $16.4 \pm 5.9$ & $4.8 \pm 3.6$ & $22.3 \pm 4.3$ & - \\
\hline $\begin{array}{l}\text { Moghaddasi, } \\
2013 \text { [126] }\end{array}$ & Iran & $\begin{array}{l}\text { Nurses working in medical and } \\
\text { education centers }(N=340)\end{array}$ & $\mathrm{MBI}$ & $\begin{array}{l}22.8 \pm 12.4 \\
34.6 \%\end{array}$ & $\begin{array}{l}6.99 \pm 6.23 \\
28.8 \%\end{array}$ & $\begin{array}{l}32.30 \pm 9.26 \\
95.7 \%\end{array}$ & - \\
\hline $\begin{array}{l}\text { Mohammad, } \\
2012[127]\end{array}$ & Iran & Nurses $(n=712)$ & $\mathrm{MBI}$ & - & - & - & $21.9 \%^{+}$ \\
\hline $\begin{array}{l}\text { Mudallal, } 2017 \\
{[128]}\end{array}$ & Jordan & Nurses $(N=407)$ & $\mathrm{MBI}$ & $\begin{array}{l}31.5 \pm 12.8 \\
60.9 \%\end{array}$ & $\begin{array}{l}15.2 \pm 6.9 \\
65.1 \%\end{array}$ & $\begin{array}{l}32.3 \pm 18.9 \\
43.0 \%\end{array}$ & - \\
\hline $\begin{array}{l}\text { Naveri, } 2009 \\
\text { [129] }\end{array}$ & Iran & Nurses $(N=200)$ & $\mathrm{MBI}$ & - & - & - & $21.7 \%^{+}$ \\
\hline $\begin{array}{l}\text { Ozbas, } 2016 \\
{[130]}\end{array}$ & Turkey & Oncology nurses $(N=82)$ & $\mathrm{MBI}$ & $\begin{array}{l}\text { Pre- } \\
\text { intervention: } \\
\text { Study group: } \\
25.3 \pm 6.8 \\
\text { Control } \\
\text { group: } \\
23.6 \pm 6.9 \\
\text { Post- } \\
\text { intervention: } \\
\text { Study group: } \\
19.0 \pm 4.0 \\
\text { Control } \\
\text { group: } \\
26.1 \pm 5.8\end{array}$ & $\begin{array}{l}\text { Pre- } \\
\text { intervention: } \\
\text { Study group: } \\
10.8 \pm 4.1 \\
\text { Control } \\
\text { group: } \\
9.7 \pm 3.0 \\
\text { Post- } \\
\text { intervention: } \\
\text { Study group: } \\
7.9 \pm 2.6 \\
\text { Control } \\
\text { group: } \\
11.1 \pm 3.1\end{array}$ & $\begin{array}{l}\text { Pre- } \\
\text { intervention: } \\
\text { Study group: } \\
29.1 \pm 4.6 \\
\text { Control } \\
\text { group: } \\
28.4 \pm 4.1 \\
\text { Post- } \\
\text { intervention: } \\
\text { Study group: } \\
30.6 \pm 3.7 \\
\text { Control } \\
\text { group: } \\
27.7 \pm 4.6\end{array}$ & - \\
\hline $\begin{array}{l}\text { Ozden, } 2013 \\
\text { [131] }\end{array}$ & Turkey & Intensive care nurses $(N=138)$ & $\mathrm{MBI}$ & $15.8 \pm 7.2$ & $6.5 \pm 4.2$ & $20.7 \pm 5.0$ & - \\
\hline $\begin{array}{l}\text { Özgür, } 2018 \\
\text { [132] }\end{array}$ & Turkey & Nurses at a university hospital $(N=155)$ & $\mathrm{MBI}$ & $20.3 \pm 5.9$ & $7.2 \pm 3.4$ & $12.76 \pm 3.67$ & - \\
\hline $\begin{array}{l}\text { Palazoglu, } \\
2017 \text { [133] }\end{array}$ & Turkey & Emergency nurses $(N=236)$ & MBI & $21.9 \pm 6.5$ & $10.0 \pm 4.0$ & $21.0 \pm 4.5$ & - \\
\hline $\begin{array}{l}\text { Rezaei, } 2018 \\
{[134]}\end{array}$ & Iran & Nurses $(N=200)$ & MBI-HSS & $2.9 \pm 0.3$ & $2.9 \pm 0.2$ & $2.6 \pm 0.5$ & - \\
\hline $\begin{array}{l}\text { Ron, } 2014 \\
{[135]}\end{array}$ & Israel & Nurses $(N=214)$ & single-item & - & - & - & $3.92 \pm 0.44$ \\
\hline $\begin{array}{l}\text { Sabanciogullari } \\
2015[136]\end{array}$ & Turkey & $\begin{array}{l}\text { Nurses working at a university hospital } \\
(N=63)\end{array}$ & $\mathrm{MBI}$ & $14.8 \pm 5.1$ & $24.6 \pm 3.7$ & $21.6 \pm 2.4$ & - \\
\hline $\begin{array}{l}\text { Sahraian, } 2008 \\
\text { [137] }\end{array}$ & Iran & $\begin{array}{l}\text { Nurses working at public hospitals } \\
(N=180)\end{array}$ & $\mathrm{MBI}$ & $25.8 \pm 0.9$ & $5.9 \pm 0.3$ & $29.6 \pm 1.1$ & - \\
\hline $\begin{array}{l}\text { Shahriari, } 2014 \\
\text { [138] }\end{array}$ & Iran & Critical care nurses $(N=170)$ & $\mathrm{MBI}$ & $\begin{array}{l}\text { Fixed shift } \\
\text { schedule: } \\
60 \% \\
\text { Non-fixed } \\
\text { schedule: } \\
12.9 \%\end{array}$ & $\begin{array}{l}\text { Fixed shift } \\
\text { schedule: } \\
32.9 \%, \\
\text { Non-fixed } \\
\text { schedule: } \\
18.8 \%\end{array}$ & $\begin{array}{l}\text { Fixed shift } \\
\text { schedule: } \\
27.1 \% \\
\text { Non-fixed } \\
\text { schedule: } \\
43.5 \%\end{array}$ & - \\
\hline $\begin{array}{l}\text { Shamali, } 2015 \\
\text { [139] }\end{array}$ & Iran & $\begin{array}{l}\text { Nurses with rotating shift schedules } \\
(N=130) \text { and fixed shift schedules } \\
(N=130)\end{array}$ & $\mathrm{MBI}$ & $\begin{array}{l}\text { Rotating } \\
\text { schedule } \\
20.5 \pm 11.1 \\
\text { Fixed } \\
\text { schedule: } \\
26.6 \pm 11.4\end{array}$ & $\begin{array}{l}\text { Rotating } \\
\text { schedule } \\
10.8 \pm 6.7 \\
\text { Fixed } \\
\text { schedule: } \\
11.7 \pm 6.4\end{array}$ & $\begin{array}{l}\text { Rotating } \\
\text { schedule } \\
31.1 \pm 9.9 \\
\text { Fixed } \\
\text { schedule: } \\
29.3 \pm 11.2\end{array}$ & - \\
\hline Sorour, 2012 & Egypt & Emergency nurses $(N=58)$ & $\mathrm{MBI}$ & - & - & - & $37.9 \% *$ \\
\hline
\end{tabular}


Table 2 Characteristics of studies on burnout among nurses in Middle East $(N=53)$ (Continued)

\begin{tabular}{|c|c|c|c|c|c|c|c|}
\hline $\begin{array}{l}\text { 1st author, } \\
\text { Year }\end{array}$ & Country & Study population & $\begin{array}{l}\text { Burnout } \\
\text { assessment }\end{array}$ & $\begin{array}{l}\mathrm{EE}(\%) \text { or } \\
\text { mean } \pm \mathrm{SD}\end{array}$ & $\begin{array}{l}\mathrm{DP}(\%) \text { or } \\
\text { mean } \pm \mathrm{SD}\end{array}$ & $\begin{array}{l}\mathrm{PA}(\%) \text { or } \\
\text { mean } \pm \mathrm{SD}\end{array}$ & $\begin{array}{l}\text { Overall } \\
\text { Burnout (\%) } \\
\text { or mean } \pm \\
\text { SD }\end{array}$ \\
\hline $\begin{array}{l}\text { Soroush, } 2016 \\
\text { [141] }\end{array}$ & Iran & $\begin{array}{l}\text { Nurses working in neonatal intensive } \\
\text { care units }(N=86)\end{array}$ & $\mathrm{MBI}$ & $21.3 \pm 8.1$ & $2.6 \pm 31$ & $22.6 \pm 5.4$ & - \\
\hline $\begin{array}{l}\text { Taleghani, } \\
2017[142]\end{array}$ & Iran & Oncology nurses $(N=67)$ & $\mathrm{MBI}$ & $38.1 \pm 22.7$ & $25.6 \pm 17.8$ & $47.9 \pm 13.7$ & - \\
\hline $\begin{array}{l}\text { Tekindal, } 2012 \\
\text { [143] }\end{array}$ & Turkeys & $\begin{array}{l}\text { Nurses working at a state hospital } \\
(N=225)\end{array}$ & $\mathrm{MBI}$ & $27.2 \pm 6.3$ & $9.3 \pm 3.1$ & $29.4 \pm 4.2$ & - \\
\hline $\begin{array}{l}\text { Topbas, } 2019 \\
{[144]}\end{array}$ & Turkey & Hemodialysis nurses $(\mathrm{N}=82$ ) & $\mathrm{MBI}$ & $15.1 \pm 7.9$ & $8.2 \pm 5.1$ & $22.3 \pm 4.9$ & \\
\hline $\begin{array}{l}\text { Tuna, } 2014 \\
{[145]}\end{array}$ & Turkey & Oncology nurses $(N=189)$ & $\mathrm{MBI}$ & $18.3 \pm 6.2$ & $5.9 \pm 3.6$ & $11.1 \pm 4.1$ & - \\
\hline $\begin{array}{l}\text { Yousefy, } 2006 \\
{[146]}\end{array}$ & Iran & $\begin{array}{l}\text { Psychiatric nurses }(N=55) \text { and } \\
\text { medical nurses }(N=51)\end{array}$ & $\mathrm{MBI}$ & $16.64 \pm 7.54$ & $4.96 \pm 5.50$ & $13.82 \pm 9.83$ & - \\
\hline
\end{tabular}

Abbreviations: $\mathrm{MBI}=$ Maslach Burnout Inventory; $M B I-H S S=$ Maslach Burnout Inventory - Human Services Survey

*Overall burnout prevalence based on high burnout score in at least one of the burnout categories

$\wedge$ Overall burnout prevalence based on high burnout scores in all three of the burnout categories

+Unclear how overall burnout prevalence was defined

was 67.1 and $56.5 \%$ respectively $[167,168]$. A study in Iran found that among 230 medical students, burnout scores were high for emotional exhaustion (mean \pm SD: $20.2 \pm 10.1)$, depersonalization $(6.6 \pm 5.3)$, and personal accomplishment $(34.9 \pm 8.6,169)$. Among 165 medical students in Lebanon, $75 \%$ were reported to exhibit high levels of overall burnout [170]. Another study in Lebanon found that $43 \%$ of students $(N=176)$ were experiencing high levels of overall burnout according to the Burnout Measure [171]. Finally, among 302 medical students in Turkey, high burnout scores were reported for emotional exhaustion (25.5 \pm 7.5$)$, depersonalization (11.3 \pm 3.9$)$, and personal accomplishment $(24.7 \pm 3.4)$ [172].

\section{Risk factors associated with burnout among healthcare providers}

Components in the work environment were statistically significantly associated with measures of burnout, including heavy workload, unorganized and difficult working conditions, difficulties balancing professional and private life, and income. For example, among medical residents in Lebanon $(N=155)$, those who reported working more than $80 \mathrm{~h}$ a week were 2.91 times as likely to report burn out compared to residents working less than $80 \mathrm{~h}$ a week [52]. In a Saudi Arabia study conducted among primary care physicians $(N=246)$, multivariate regression analysis showed that the number of patients per day $(p<0.001)$, more paperwork $(\mathrm{p}<0.001)$, unorganized patients flow to clinics $(p=0.021)$, and patient pressure/violence $(\mathrm{p}<0.001)$ were all statistically significant positive predictors of burnout [82]. Work overload and difficulties balancing professional and private life were both reported to be significant predictors of high emotional exhaustion $(p<0.05)$ in a study done in Iran [62]. A study in Turkey found among assistant physicians working at a university hospital $(N=222)$, those who considered their monthly income level as "poor" differed significantly from those who regarded their monthly income levels as "good" in terms of mean scores in subscales of the MBI, emotional exhaustion, depersonalization, and personal accomplishment $(\mathrm{p}<0.05)$ [70]. A study in Egypt among 227 nurses in a university hospital reported that the number of shifts and shift timing (night) proved to be significant predictors for high grades of the three domains of burnout collectively among nurses [100]. Among 333 nurses at university and state hospitals in Turkey, always working night was found the be positively associated with all subscales of burnout as reported by the MBI $(\mathrm{p}<0.05)$ as compared to always working day shift or occasionally working night shift [107]. A study in Iran of critical care nurses $(N=170)$ reported that among nurses with fixed shift schedules, the odds of high risk of emotional exhaustion as measured by the MBI was 10.1 (95\%CI: 4.68-21.75) times higher than the odds among nurses with non-fixed shift schedule [135].

Exposure to violence, terror, and conflict were all associated with increased odds of burnout. This suggests there may be an interaction between burnout and secondary traumatic stress in Middle East caregivers. The war trauma primary and secondary exposure as being worsening factors in burnout was specifically mentioned and reported in a study related to Lebanese nurses working in academic centers. The study also linked trauma exposure to higher level of depression in nurses (36.2\%) compared to $10 \%$ of general population [171]. Moreover, among emergency physicians working in Turkey $(N=174)$, there were significant associations 
Table 3 Characteristics of studies on burnout among combined populations of healthcare workers in the Middle East ( $\mathrm{N}=22$ )

\begin{tabular}{|c|c|c|c|c|c|c|c|}
\hline 1st author, Year & Country & Study population & $\begin{array}{l}\text { Burnout } \\
\text { assessment }\end{array}$ & $\begin{array}{l}\mathrm{EE}(\%) \text { or } \\
\text { mean } \pm \mathrm{SD}\end{array}$ & $\begin{array}{l}\mathrm{DP}(\%) \text { or } \\
\text { mean } \pm \mathrm{SD}\end{array}$ & $\begin{array}{l}\mathrm{PA}(\%) \text { or } \\
\text { mean } \pm \text { SD }\end{array}$ & $\begin{array}{l}\text { Overall } \\
\text { Burnout or } \\
\text { mean } \pm S D\end{array}$ \\
\hline $\begin{array}{l}\text { Abarghouei, } \\
2016[147]\end{array}$ & Iran & $\begin{array}{l}\text { Hospital personnel: }(N=340) \text { : health sector } \\
(N=170) \text { and administrative sector }(N=170)\end{array}$ & $\mathrm{MBI}$ & $21.7 \pm 7.3$ & $9.6 \pm 3.7$ & $26.8 \pm 6.2$ & - \\
\hline $\begin{array}{l}\text { Abdo, } 2016 \\
{[148]}\end{array}$ & Egypt & $\begin{array}{l}\text { Physicians }(N=266) \text { and nurses }(N=284) \\
\text { at an emergency hospital }\end{array}$ & $\mathrm{MBI}$ & $\begin{array}{l}\text { Nurses: } \\
52.8 \% \\
\text { Physicians: } \\
29.7 \%\end{array}$ & $\begin{array}{l}\text { Nurses: } \\
44.4 \% \\
\text { Physicians: } \\
45.6 \%\end{array}$ & $\begin{array}{l}\text { Nurses: } 96.5 \% \\
\text { Physicians: } \\
99.2 \%\end{array}$ & - \\
\hline $\begin{array}{l}\text { Alacacioglu, } \\
2009 \text { [149] }\end{array}$ & Turkey & $\begin{array}{l}\text { Physicians }(N=77) \text { and nurses }(N=56) \text { at an } \\
\text { oncology clinic }\end{array}$ & $\mathrm{MBI}$ & $\begin{array}{l}\text { Nurses: } \\
5.4 \% \\
\text { Physicians: } \\
7.8 \%\end{array}$ & $\begin{array}{l}\text { Nurses: } \\
5.4 \% \\
\text { Physicians: } \\
15.6 \%\end{array}$ & $\begin{array}{l}\text { Nurses: } 100 \% \\
\text { Physicians: } \\
\text { 100\% }\end{array}$ & - \\
\hline $\begin{array}{l}\text { Alameddine, } \\
2011[150]\end{array}$ & Lebanon & $\begin{array}{l}\text { Employees working at an emergency department } \\
(N=256) \text { : }\end{array}$ & $\mathrm{MBI}$ & $54.9 \%$ & $43.5 \%$ & $44.5 \%$ & - \\
\hline $\begin{array}{l}\text { Alameddine, } \\
2012 \text { [151] }\end{array}$ & Lebanon & Health providers $(N=755)$ & $\mathrm{MBI}$ & $23.3 \%$ & $12.8 \%$ & $18.7 \%$ & - \\
\hline $\begin{array}{l}\text { Alameddine, } \\
2017 \text { [152] }\end{array}$ & Lebanon & Health providers $(N=1000)$ & MBI-HSS & $22.1 \%$ & $10.8 \%$ & $42.1 \%$ & - \\
\hline $\begin{array}{l}\text { Ashtari, } 2009 \\
{[153]}\end{array}$ & Iran & Mental health service providers $(\mathrm{N}=100)$ & $\mathrm{MBI}$ & $29.4 \pm 6.9$ & $9.3 \pm 2.1$ & $35.5 \pm 7.0$ & - \\
\hline $\begin{array}{l}\text { Bijari, } 2016 \\
{[154]}\end{array}$ & Iran & Rural health workers $(N=423)$ & $\mathrm{MBI}$ & $17.7 \%$ & $6.4 \%$ & $53 \%$ & $5.7 \% \wedge$ \\
\hline $\begin{array}{l}\text { Calgan, } 2011 \\
{[155]}\end{array}$ & Turkey & Community pharmacists $(N=251)$ & $\mathrm{MBI}$ & 16.8 & 4.0 & 22.0 & - \\
\hline $\begin{array}{l}\text { Demirci, } 2010 \\
\text { [156] }\end{array}$ & Turkey & Oncology employees $(N=90)$ & $\mathrm{MBI}$ & $\begin{array}{l}23.80 \pm \\
10.98 \\
42 \%\end{array}$ & $\begin{array}{l}5.21 \pm 4.99 \\
20 \%\end{array}$ & $\begin{array}{l}36.23 \pm 8.05 \\
35.6 \%\end{array}$ & - \\
\hline $\begin{array}{l}\text { Devebakan, } \\
2018 \text { [157] }\end{array}$ & Turkey & Healthcare workers $(N=80)$ & $\mathrm{MBI}$ & - & - & - & - \\
\hline $\begin{array}{l}\text { Gokcen, } 2013 \\
\text { [158] }\end{array}$ & Turkey & $\begin{array}{l}\text { Emergency department workers }(N=270) \text { : } \\
\text { doctors }(N=630) \text {, nurses }(N=85) \text {, paramedics ( } N= \\
\text { 122) }\end{array}$ & $\mathrm{MBI}$ & $14.3 \pm 8.2$ & $6.5 \pm 4.3$ & $18.9 \pm 6.5$ & \\
\hline $\begin{array}{l}\text { Gulalp, } 2008 \\
\text { [159] }\end{array}$ & Israel & $\begin{array}{l}\text { Emergency physicians ( } N=8) \text { nurses }(N=40) \text {, and } \\
\text { nurse's aide }(N=12)\end{array}$ & $\mathrm{MBI}$ & $19.1 \pm 9.1$ & $7.8 \pm 4.7$ & $22.3 \pm 5.9$ & - \\
\hline $\begin{array}{l}\text { Guveli, } 2015 \\
\text { [160] }\end{array}$ & Turkey & Oncology health workers $(N=159)$ & $\mathrm{MBI}$ & $14.2 \pm 7.2$ & $4.9 \pm 3.4$ & $6.2 \pm 5.3$ & - \\
\hline $\begin{array}{l}\text { Hamdan, } 2017 \\
\text { [161] }\end{array}$ & Palestine & $\begin{array}{l}\text { Emergency workers: physicians }(N=142) \text {, nurses } \\
(N=161) \text {, and administrative personnel }(N=141) \text {. }\end{array}$ & MBI-HSS & $64.8 \%$ & $38.1 \%$ & $34.6 \%$ & - \\
\hline $\begin{array}{l}\text { Hosseiniarzfuni, } \\
2015 \text { [162] }\end{array}$ & Iran & Nurses $(N=35)$ and technicians $(N=32)$ & $\mathrm{MBI}$ & - & - & - & $\begin{array}{l}\text { Technicians: } \\
\text { 19\%^ } \\
\text { Nurses: } \\
24 \% \wedge\end{array}$ \\
\hline $\begin{array}{l}\text { Kabir, } 2016 \\
{[163]}\end{array}$ & Iran & Healthcare workers $(N=1141)$ & $\mathrm{MBI}$ & - & - & - & $9.1 \% \wedge$ \\
\hline $\begin{array}{l}\text { Kömür } 2017 \\
{[164]}\end{array}$ & Turkey & $\begin{array}{l}\text { Mortuary staff }(N=142) \text { : forensic medicine } \\
\text { specialists }(N=40) \text {, forensic medicine residents } \\
(N=54) \text {, autopsy technicians }(N=45) \text { and other } \\
\text { staff members }(N=24)\end{array}$ & $\mathrm{MBI}$ & $\begin{array}{l}11.1 \pm 6.6 \\
14 \%\end{array}$ & $\begin{array}{l}8.2 \pm 3.6 \\
32.4 \%\end{array}$ & $\begin{array}{l}17.4 \pm 5.5 \\
76.1 \%\end{array}$ & - \\
\hline $\begin{array}{l}\text { Malakouti, } 2011 \\
{[165]}\end{array}$ & Iran & Rural mental health workers (Behvarzes) $(N=212)$ & $\mathrm{MBI}$ & $14.5 \pm 9.9$ & $2.2 \pm 3.4$ & $33.8 \pm 10.4$ & - \\
\hline $\begin{array}{l}\text { Tarcan, } 2017 \\
{[166]}\end{array}$ & Turkey & $\begin{array}{l}\text { Health professionals }(N=250) \text { : doctors }(N=38) \text {, } \\
\text { nurses }(N=55) \text {, technicians }(N=118) \text {, and medical } \\
\text { secretaries }(N=39) \text {. }\end{array}$ & MBI-HSS & $3.6 \pm 1.3$ & $2.8 \pm 1.2$ & $3.8 \pm 1.1$ & - \\
\hline $\begin{array}{l}\text { Tekin, } 2017 \\
{[167]}\end{array}$ & Turkey & $\begin{array}{l}\text { Health care professionals }(N=120) \text { : nurses }(N=73) \\
\text { and doctors }(N=47)\end{array}$ & $\mathrm{MBI}$ & $\begin{array}{l}\text { Type D } \\
\text { personality } \\
\text { (negative): } \\
20.7 \pm 7.6\end{array}$ & $\begin{array}{l}\text { Type D } \\
\text { personality } \\
\text { (negative): } \\
6.8 \pm 4.2\end{array}$ & $\begin{array}{l}\text { Type D } \\
\text { personality } \\
\text { (negative): } \\
11.7 \pm 5.2\end{array}$ & - \\
\hline
\end{tabular}


Table 3 Characteristics of studies on burnout among combined populations of healthcare workers in the Middle East ( $\mathrm{N}=22$ ) (Continued)

\begin{tabular}{|c|c|c|c|c|c|c|c|}
\hline 1st author, Year & Country & Study population & $\begin{array}{l}\text { Burnout } \\
\text { assessment }\end{array}$ & $\begin{array}{l}\mathrm{EE}(\%) \text { or } \\
\text { mean } \pm \mathrm{SD}\end{array}$ & $\begin{array}{l}\mathrm{DP}(\%) \text { or } \\
\text { mean } \pm \mathrm{SD}\end{array}$ & $\begin{array}{l}\mathrm{PA}(\%) \text { or } \\
\text { mean } \pm \mathrm{SD}\end{array}$ & $\begin{array}{l}\text { Overall } \\
\text { Burnout or } \\
\text { mean } \pm S D\end{array}$ \\
\hline & & & & $\begin{array}{l}\text { (positive): } \\
16.3 \pm 8.7\end{array}$ & $\begin{array}{l}\text { (positive): } \\
4.5 \pm 4.2\end{array}$ & $\begin{array}{l}\text { (positive): } \\
9.7 \pm 5.9\end{array}$ & \\
\hline $\begin{array}{l}\text { Tunc, } 2009 \\
{[168]}\end{array}$ & Turkey & $\begin{array}{l}\text { Health care professionals (250): physicians } \\
(N=170) \text { and nurses }(N=81)\end{array}$ & MBI-HSS & $\begin{array}{l}\text { Physicians: } \\
1.6 \pm 0.9 \\
\text { Nurses: } \\
2.2 \pm 0.9\end{array}$ & $\begin{array}{l}\text { Physicians: } \\
0.9 \pm 0.7 \\
\text { Nurses: } \\
1.3 \pm 0.8\end{array}$ & $\begin{array}{l}\text { Physicians: } \\
1.0 \pm 0.7 \\
\text { Nurses: } \\
1.4 \pm 0.8\end{array}$ & - \\
\hline
\end{tabular}

Abbreviations: $\mathrm{MBI}=$ Maslach Burnout Inventory; $M B I-H S S$ = Maslach Burnout Inventory - Human Services Survey

*Overall burnout prevalence based on high burnout score in at least one of the burnout categories

$\wedge$ Overall burnout prevalence based on high burnout scores in all three of the burnout categories

+Unclear how overall burnout prevalence was defined

between emotional exhaustion, as reported by the MBI, and overall violence $(p=0.012)$ and verbal violence $(p=0.016)$ and depersonalization and total violence $(p=0.021)$ and verbal violence $(p=0.012)$ [56]. A study done on healthcare workers in Palestine $(N=444)$ reported that the odds of high risk of burnout among those who had exposure to violence was two times the odds among those who had no exposure to violence [158]. Additionally, a study in Israel which assessed burnout among physicians in conflict zones $(N=$ 97) reported that higher levels of PTSD symptoms due to war and terror were associated with higher levels of compassion fatigue $(\beta=0.594 ; \mathrm{t}=4.419 ; p<0.001)$ [85]. Another study in Israel among nurses $(N=214)$ reported that nurses' exposure to national terror and the level of distress they experienced due to ongoing terror attacks were statistically significantly associated with high levels of burnout [132].

Reports examining the association between age, time in career, and burnout were variable. Overall, results suggest that the association between age and burnout is bimodal, in which younger age (i.e. under 25) and old age (i.e. over 55), along with greater work experience are associated with the highest burnout levels. Many studies suggested that younger age was a significant predictor of burnout. For example, a study among physicians in Saudi Arabia $(N=270)$ found that younger age was significantly associated with higher emotional exhaustion and lower personal accomplishment scores [44]. A study in Turkey found that among physicians $(N=663)$, mean emotional exhaustion and depersonalization scores were significantly higher and personal accomplishment scores were significantly lower in participants aged under 25 years [66]. Among two different studies of nurses in Turkey $(N=141)$ and $(N=171)$ younger age was also found to be associated with increased risk of burnout $[98,118]$. Among burn clinicians in Israel $(N=55)$, older age was found to be a protective factor for high risk of overall burnout ( $\mathrm{OR}=0.79, p=0.019)$ [59]. Among oncology employees $(N=90)$ working in Turkey, being of

Table 4 Characteristics of studies on burnout among medical students in the Middle East $(N=7)$

\begin{tabular}{|c|c|c|c|c|c|c|c|}
\hline $\begin{array}{l}\text { First Author, } \\
\text { Year }\end{array}$ & Country & Study population & $\begin{array}{l}\text { Burnout } \\
\text { assessment }\end{array}$ & $\begin{array}{l}\mathrm{EE}(\%) \text { or } \\
\text { mean } \pm \mathrm{SD}\end{array}$ & $\begin{array}{l}\text { DP }(\%) \text { or } \\
\text { mean } \pm S D\end{array}$ & $\begin{array}{l}\mathrm{PA}(\%) \text { or } \\
\text { mean } \pm \mathrm{SD}\end{array}$ & $\begin{array}{l}\text { Overall Burnout or } \\
\text { mean } \pm S D\end{array}$ \\
\hline $\begin{array}{l}\text { Al-Alawi, } 2017 \\
\text { [169] }\end{array}$ & Oman & $\begin{array}{l}\text { Medical students } \\
(N=662)\end{array}$ & $\mathrm{MBI}$ & $30.1 \%$ & $33.9 \%$ & $31.1 \%$ & $7.4 \% \wedge$ \\
\hline $\begin{array}{l}\text { Almalki, } 2017 \\
{[170]}\end{array}$ & $\begin{array}{l}\text { Saudi } \\
\text { Arabia }\end{array}$ & $\begin{array}{l}\text { Medical students } \\
(N=249)\end{array}$ & MBI-HSS & $62.2 \%$ & $58.6 \%$ & $60.2 \%$ & $67.1 \% *$ \\
\hline $\begin{array}{l}\text { Altannir, } 2019 \\
{[171]}\end{array}$ & $\begin{array}{l}\text { Saudi } \\
\text { Arabia }\end{array}$ & $\begin{array}{l}\text { Medical students } \\
(N=276)\end{array}$ & $\mathrm{MBI}$ & $\begin{array}{l}18.5 \pm 10.3 \\
17.4 \%\end{array}$ & $\begin{array}{l}14.2 \pm 9.2 \\
56.9 \%\end{array}$ & $\begin{array}{l}28.7 \pm 9.5 \\
14.9 \%\end{array}$ & $13.4 \wedge$ \\
\hline $\begin{array}{l}\text { Ebrahimi, } 2018 \\
\text { [172] }\end{array}$ & Iran & $\begin{array}{l}\text { Medical students } \\
(\mathrm{N}=230)\end{array}$ & $\mathrm{MBI}$ & $20.2 \pm 10.2$ & $6.7 \pm 5.3$ & $34.9 \pm 8.6$ & - \\
\hline Fares, 2016 [173] & Lebanon & $\begin{array}{l}\text { Medical students } \\
(N=165)\end{array}$ & MBI-HSS & - & - & - & $75 \%^{+}$ \\
\hline $\begin{array}{l}\text { Sevencan, } 2010 \\
{[175]}\end{array}$ & Turkey & $\begin{array}{l}\text { Medical students } \\
(N=302)\end{array}$ & $\mathrm{MBI}$ & $25.5 \pm 7.5$ & $11.3 \pm 3.9$ & $24.7 \pm 3.4$ & - \\
\hline Talih, 2018 [174] & Lebanon & $\begin{array}{l}\text { Medical students } \\
(N=176)\end{array}$ & $\begin{array}{l}\text { Burnout } \\
\text { Measure }\end{array}$ & - & - & - & $43 \%$ \\
\hline
\end{tabular}

Abbreviations: $M B I$ = Maslach Burnout Inventory; MBI-HSS = Maslach Burnout Inventory - Human Services Survey

*Overall burnout prevalence based on high burnout score in at least one of the burnout categories

$\wedge$ Overall burnout prevalence based on high burnout scores in all three of the burnout categories

${ }^{+}$Unclear how overall burnout prevalence was defined 
age less than 35 years was significantly associated with burnout [153]; however, this study also reported that work experience greater than 10 years was also significantly associated with burnout. In that same vein, other studies reported that increasing age and greater occupational experience was positively associated with burnout. Among psychiatric trainees $(N=167)$ in Turkey, logistic regression confirmed that older age $(p=0.02)$ was associated with severe burnout [55]. Among nurses in Iran $(N=200)$, greater age and work experience accounted for $30 \%$ of the variance in depersonalization [131]. A study in Egypt among 266 physicians and 284 nurses also found that age and years of experience were significant predictors of burnout [145]. In Iran among 1141 healthcare workers, the number of years of experience in the health professional was also associated with increased levels of burnout $(p<0.001)$ [160].

The majority of studies that found a significant association between gender and burnout reported that female gender was a significant predictor of increased risk for burnout $[38,48,52,81,89,103,105,117,125,163,165$, 168, 173].. For example, among trainee anesthesiologists in Turkey $(N=159)$ and physicians in Saudi Arabia $(N=$ 348), female gender was significantly associated with greater burnout scores $(p<0.05,0.02$ respectively) [38, 48]. Among nephrology nurses in Israel $(N=132)$ and nurses in Jordan $(N=407)$, female gender was also found to be a significant predictor of burnout $(\mathrm{p}<0.05, p<0.01)$ $[105,125]$. Among medical students in Saudi Arabia $(N=$ 276), female gender was a significant predictor of emotional exhaustion and depersonalization $(\mathrm{OR}=4.34, p=$ 0.001 ) [168]. Only three studies reported that males were associated with higher burnout risk compared to females: among 130 pediatricians in Saudi Arabia, the prevalence of burnout among males was significantly greater than females $(40 \%$ vs $31 \% ; p=0.012,45)$. Another study in Turkey among nurses working in dialysis centers $(N=$ 171) found that male sex was associated with higher depersonalization scores $(p<0.05)$ [118]. Another study in Iran found that among 340 hospital personnel, emotional exhaustion and depersonalization scores were higher among males compared to females [144]. Lastly, some studies reported no association at all between gender and burnout, such as a study among 302 medical students in Turkey [172] and a study among primary care physicians in Iran $(N=548)$ [50].

Experiences of stress and lack of job support were additionally found to be predictive of job burnout among healthcare professionals. For example, among trainee anesthesiologists in Turkey, perceived stress, as measured by the Perceived Stress Scale, were significantly associated with increased odds of burnout $(p<0.05)$ [38]. Among anesthesiologists in Egypt, there was a significant positive correlation between job stress, as measured by
Workplace Stress Scale of the American Institute of Stress, and MBI-HSS subscales [74]. Moreover a significant positive relationship between sub dimensions of job stress level, as measured by the Job Stressors Scale, and of burnout level $(\mathrm{p}<0.05)$ was found among oncology nurses in Turkey [142]. Furthermore, the strongest significant single predictor of all burnout dimensions among anesthesiologists in Egypt $(N=98)$ was lack of job support [74]. Among nurses from four teaching hospitals in Iran $(N=$ 684), lack of workplace support was also found to be a significant predictor of burnout $(\beta=-0.043$, CI $95 \%=$ -0.097--0.003) [103].

\section{Burnout intervention programs}

There were only a few programs aimed at alleviating burnout in the Middle East. Only three studies examined burnout-related interventions in physicians [53, 68, 83], and only four examined interventions for nurses [106, 112, 127, 133]. Bar-Sela et al. examined the effect of "Balint" group meetings as an intervention aimed at improving burnout levels among oncology junior and senior residents in Israel [53]. The oncology residents attended nine sessions in one year, in which they meet to discuss cases from the residents' experiences. From a comparison of MBI scores, the investigators found that higher measures of emotional exhaustion and depersonalization were noted in junior residents at the beginning of the year, before the intervention; however, in senior residents, all measures of burnout increased after the intervention. In Qatar, Ghannam et al. assessed the effect of a stress management intervention on residents' $(N=256)$ burnout outcomes [83]. They conducted a 1-day workshop with the objectives being to help residents identify stressors, identify early warning signs of stress, and practice intervention techniques. At 1-month post-course, $83.6 \%$ listed at least one piece of knowledge or skill that they had put into practice since the course. Using the abbreviated MBI, residents improved in three of the four burnout constructs: emotional exhaustion, depersonalization, and satisfaction with the practice of medicine. Kotb, Mohamed, Kamel, Ismail and Abdulmajeed evaluated the effect of an educational program on level of professional burnout among family physicians working in family practice centers in Egypt $(N=31)$ [68]. Prevalence of burnout after six months of the intervention program decreased from (41.9\%) to (32.3\%).

In Iran, Darban, Balouchi, Narouipour, Sarfazaei and Shahdahi examined the effect of a communication skills training on the burnout of nurses [106]. The training was carried out for the intervention group as a 2-day workshop for $8 \mathrm{~h}$ within a week. They found that the mean score of job burnout in the intervention group significantly decreased while mean scores of burnout in the control group showed no significant change $(p=0.01)$. A 
randomized controlled trial was conducted in Turkey by Gunusen and Ustun to evaluate the effects of coping and support group interventions on the reduction of burnout among nurses [112]. Cognitive coping strategies and problem-solving methods were used for coping training. The objectives of the social support groups were to provide support, information and a sense of belonging, and create an environment where individuals share their experiences. Right after the intervention, there was an immediate reduction in emotional exhaustion dimension in the intervention group, as assessed by the MBI, of burnout. However, after 6 months, scores were increased again.

Another study in Turkey was conducted to determine the effect of a "psychodrama-based psychological empowerment program" on the levels of burnout in oncology nurses $(N=82)$ [127]. They found that there was a statistically significant difference between nurses' scores in the sub dimensions of emotional burnout, depersonalization, and personal accomplishment $(p<0.05)$, and such that nurses in the study group had lower levels of emotional burnout and desensitization and high personal achievement scores at one and three months after the psychological empowerment program compared to nurses in the control/comparison group. Lastly, Sabanciogullari and Dogan evaluated the effects of the Professional Identity Development Program on burnout levels of registered nurses in Turkey [133]. The program consisted of ten sessions delivered to the study group once a week. During the research period, burnout levels significantly decreased in the study group, while those of the control group increased.

\section{Discussion}

Burnout is prevalent among physicians, nurses, and other medical professionals in the Middle East. Prevalence estimates range between 40 and $60 \%$ with reported rates between 13 and 100\% (Tables S1-4). The minority of studies that reported lower than average levels of burnout attributed such differences to the quality of their study, sample size, and characteristics of the population studied. Nurses reported the highest levels of burnout among healthcare providers. High levels of burnout were associated with harsh work conditions, stress, and exposure to violence and conflict. Studies in the Middle East maintain previous research among healthcare providers that have demonstrated that risk factors for burnout include female gender, young age, and low support and resources to handle workload. The number of reported interventions aimed at alleviating burnout in the Middle East is scarce. Nevertheless, the interventions that have been attempted are similar to some that have shown benefits in North America and Europe (e.g cognitive skills $(178 ; 179)$, support groups (180-182), Balint groups (183; 184)). Mindfulness interventions were mentioned anecdotally in several papers that did not meet inclusion criteria for this review. The duration of these interventions varied from a week [109] to a year [56] depending on the format. Repetitive and regular interventions that raise awareness and inform professionals about burnout, emotional empowerment, and stress management may have the potential to render a long-lasting protective effect on the risk of occupational burnout in the Middle East. Therefore, it would be reasonable to encourage studies of contextualized caregiver interventions that address culturally relevant ways to reduce the toxic stress of working with traumatized populations in under-resourced settings while also enhancing resilience factors.

\section{Limitations of current studies}

We noted that the MBI was used as the assessment tool for burnout in the majority of studies. Among the studies that used the MBI, we point out that the burnout scores were presorted in multiple manners either listing the percentage of participants with high burnout on each subscale, or the percentage of participants with high burnout on each subscale and the total score, and the percentage of participants with high total burnout. Some listed the percentage of participants with high burnout on emotional subscale only or the total and individual burnout as continuous scores together or separately. Finally, some studies reported individual burnout as continuous score while others reported individual burnout as continuous scores and percentages of participants. In addition, there were three different versions of the MBI used namely: MBI, MBI-HSS, and Abbreviated MBI (or AMI) rendering difficult the direct comparison of burnout rates in different countries and populations of healthcare workers. Additionally, recent work has raised concerns about how the MBI operationalizes burnout [174]. Having said that, the MBI has not been validated in healthcare workers in the Middle East, and there may be different cultural interpretations of questions related to the construct of burnout.

In addition, Table 3 points to a total of 22 studies examining burnout in combined populations of healthcare workers in the Middle East knowing that those workers have highly variable job workload and responsibilities and should not have been lumped together for precision sake. Last but not least, the cross-sectional studies lacked temporality and varied in quality adding to the limitations of the small sample sizes. (Additional file 3: Table S3, Additional file 4: Table S4, Additional file 5: Table S5 and Additional file 6: Table S6).

\section{Conclusion}

In contrast to high-income countries, the Middle East has not given much attention to burnout, despite the 
many stressors facing its healthcare community. Given the adverse health effects of burnout on patients and providers alike, the ever-increasing burden of caring for major public health threats, amidst ongoing regional conflicts and refugee crises with a paucity of resources and shortage of support, more attention needs to be paid to healthcare provider well-being in the Middle East. Further studies should include longitudinal assessments tackling cognition, performance and quality of life as well as reports on mood, substance use, and safety issues such as suicide, to enhance our understanding of the burnout syndrome and its consequences in the Middle East. Moreover, efforts should be channeled to assessing burnout consistently with a validated instrument or developing new instruments that would perform culturally better in the context of the Middle East. Finally, designing and deploying programs aimed at raising awareness, promoting well-being and burnout prevention, and improving coping with burnout symptoms through evidence-based stress management and resiliency training in the Middle East would be the gold standard for any academic, private or public institution promoting excellence in care for the population they serve.

\section{Supplementary information}

Supplementary information accompanies this paper at https://doi.org/10. 1186/s12889-019-7713-1

\section{Additional file 1: Table S1. Preferred Reporting Items for Systematic} Review and Meta-Analyses (PRISMA) guidelines.

Additional file 2: Table S2. Database terms of search.

Additional file 3: Table S3. Quality assessment based on modified Newcastle-Ottawa Scale of studies on burnout among physicians in the Middle East $(N=54)$.

Additional file 4: Table S4. Quality assessment based on modified Newcastle-Ottawa Scale of studies on burnout among nurses in the Middle East $(N=53)$

Additional file 5: Table S5. Quality assessment based on the Newcastle-Ottawa Scale on burnout among healthcare workers in the Middle East $(N=22)$.

Additional file 6: Table S6. Quality assessment based on the NewcastleOttawa Scale on burnout among midwives, medical and nursing students in the Middle East $(N=7)$

\section{Abbreviations}

AMI: Abbreviated Maslach Inventory; MBI: Maslach Burnout Inventory; MBIHSS: Maslach Burnout Inventory - Human Services Survey; PRISMA: Preferred Reporitng Items for Systematic Review and Meta-Analyses;

ProQOL: Professional Quality of Life; SCT: Sluggish Cognitive Tempo; UAE: United Arab Emirates

\section{Acknowledgements}

Not applicable.

\section{Author's contributions}

ZC, FE, and BG conceived and designed the review. ZC and FE performed the literature search. $F E, Z C, B G, M D, J S, M B, B D$, and GF contributed to the writing and editing of the manuscript. ZC, FE, BG, MD, JS, MB, BD, and GF read and approved the final manuscript.

\section{Funding}

MLD was supported by K23AT009218 from the National Center for Complementary and Integrative Health at the National Institutes of Health $(\mathrm{NIH})$. The $\mathrm{NIH}$ had no role in study design; in the collection, analysis and interpretation of data; in the writing of the report; and in the decision to submit the paper for publication.

\section{Availability of data and materials}

Not applicable.

Ethics approval and consent to participate

Not applicable.

\section{Consent for publication}

Not applicable.

\section{Competing interests}

The authors have no competing interests to declare.

\section{Author details}

${ }^{1}$ Departments of Neurology and Psychiatry, Massachusetts General Hospital, Boston, MA, USA. ${ }^{2}$ Department of Epidemiology, Harvard T.H. Chan School of Public Health, Boston, MA, USA. ${ }^{3}$ The Chester M. Pierce, MD Division of Global Psychiatry, Massachusetts General Hospital, Boston, MA, USA. ${ }^{4}$ Benson-Henry Institute for Mind Body Medicine and, Division of General Internal Medicine, Massachusetts General Hospital, Boston, MA, USA. ${ }^{5}$ Department of Neurology, American University of Beirut Medical Center, Beirut, Lebanon. ${ }^{6}$ Department of Psychiatry, American University of Beirut Medical Center, Beirut, Lebanon. 7 Department of Psychiatry, Addis Ababa University, College of Health Sciences, Addis Ababa, Ethiopia. ${ }^{8}$ Department of Psychiatry, Benson-Henry Institute for Mind Body Medicine, Massachusetts General Hospital, Boston, MA, USA. ${ }^{9}$ Harvard Medical School, Boston, MA, USA.

Received: 29 May 2019 Accepted: 30 September 2019

Published online: 22 October 2019

\section{References}

1. Maslach C, Jackson SE. The measurement of experienced burnout. J Organ Behav. 1981;2(2):99-113.

2. Bender A, Farvolden P. Depression and the workplace: a progress report. (1535-1645 (Electronic)).

3. Morse G, Salyers Mp Fau - Rollins AL, Rollins Al Fau - Monroe-DeVita M, Monroe-DeVita M Fau - Pfahler C, Pfahler C. Burnout in mental health services: a review of the problem and its remediation. (1573-3289 (Electronic))

4. Gelsema TI, van der Doef M Fau - Maes S, Maes S Fau - Janssen M, Janssen M Fau - Akerboom S, Akerboom S Fau - Verhoeven C, Verhoeven C. A longitudinal study of job stress in the nursing profession: causes and consequences. (0966-0429 (Print)).

5. Shanafelt TD, Hasan O, Dyrbye LN, Sinsky C, Satele D, Sloan J, et al. Changes in Burnout and Satisfaction With Work-Life Balance in Physicians and the General US Working Population Between 2011 and 2014. (1942-5546 (Electronic))

6. M. H. Survey of America's Physicians: Practice Patterns \& Perspectives. https:// physiciansfoundationorg/wp-content/uploads/2018/09/physicianssurvey-results-final-2018pdf. 2018.

7. Frajerman A, Morvan Y, Krebs MO, Gorwood P, Chaumette B. Burnout in medical students before residency: a systematic review and meta-analysis. Eur Psychiat. 2019;55:36-42.

8. Rodrigues H, Cobucci R, Oliveira A, Cabral JV, Medeiros L, Gurgel K, et al. Burnout syndrome among medical residents: a systematic review and metaanalysis. PLoS One. 2018;13(11):e0206840.

9. Molina-Praena J, Ramirez-Baena L, Gomez-Urquiza JL, Canadas GR, De la Fuente El, Canadas-De la Fuente GA. Levels of Burnout and Risk Factors in Medical Area Nurses: A Meta-Analytic Study. Int J Environ Res Public Health. 2018;15(12).

10. Soler JK, Yaman H, Esteva M, Dobbs F, Asenova RS, Katić M, et al. Burnout in European family doctors: the EGPRN study. Fam Pract. 2008;25(4):245-65.

11. Wu S, Singh-Carlson S, Odell A, Reynolds G, Su Y. Compassion fatigue, burnout, and compassion satisfaction among oncology nurses in the United States and Canada. Oncol Nurs Forum. 2016;43(4):E161-9. 
12. Sharma A, Sharp DM, Walker LG, Monson JR. Stress and burnout in colorectal and vascular surgical consultants working in the UK National Health Service. Psycho-oncology. 2008;17(6):570-6.

13. Aiken LH, Clarke SP, Sloane DM, Sochalski J, Silber JH. Hospital nurse staffing and patient mortality, nurse burnout, and job dissatisfaction. Jama. 2002 288(16):1987-93.

14. Gundersen L. Physician burnout. Ann Intern Med. 2001;135(2):145-8.

15. Parker PA, Kulik JA. Burnout, self- and supervisor-rated job performance, and absenteeism among nurses. J Behav Med. 1995;18(6):581-99.

16. Shanafelt TD, Bradley KA, Wipf JE, Back AL. Burnout and self-reported patient care in an internal medicine residency program. Ann Intern Med. 2002;136(5):358-67.

17. Trufelli DC, Bensi CG, Garcia JB, Narahara JL, Abrao MN, Diniz RW, et al. Burnout in cancer professionals: a systematic review and meta-analysis. Eur J Cancer Care (Engl). 2008;17(6):524-31.

18. Dyrbye LN, West CP, Satele D, Boone S, Tan L, Sloan J, et al. Burnout among U.S. medical students, residents, and early career physicians relative to the general U.S. population. Acad Med. 2014;89(3):443-51.

19. Maslach C, Leiter MP. Early predictors of job burnout and engagement. J Appl Psychol. 2008;93(3):498-512.

20. Eckleberry-Hunt J, Lick D, Boura J, Hunt R, Balasubramaniam M, Mulhem E, et al. An exploratory study of resident burnout and wellness. Acad Med. 2009;84(2):269-77.

21. Salvagioni DAJ, Melanda FN, Mesas AE, Gonzalez AD, Gabani FL, Andrade SM. Physical, psychological and occupational consequences of job burnout: a systematic review of prospective studies. PLoS One. 2017;12(10):e0185781

22. Williams ES, Konrad TR, Scheckler WE, Pathman DE, Linzer M, McMurray JE, et al. Understanding physicians' intentions to withdraw from practice: the role of job satisfaction, job stress, mental and physical health. Health Care Manag Rev. 2001;26(1):7-19.

23. Rathert C, Williams ES, Linhart H. Evidence for the quadruple aim: a systematic review of the literature on physician burnout and patient outcomes. Med Care. 2018;56(12):976-84.

24. Dewa CS, Jacobs P, Thanh NX, Loong D. An estimate of the cost of burnout on early retirement and reduction in clinical hours of practicing physicians in Canada. BMC Health Serv Res. 2014;14:254.

25. Dewa CS, Loong D, Bonato S, Thanh NX, Jacobs P. How does burnout affect physician productivity? A systematic literature review. BMC Health Serv Res. 2014;14(1):325

26. Epp K. Burnout in critical care nurses: a literature review. Dynamics (Pembroke, Ont). 2012;23(4):25-31.

27. Khamisa N, Peltzer $K$, Oldenburg B. Burnout in relation to specific contributing factors and health outcomes among nurses: a systematic review. Int J Environ Res Public Health. 2013;10(6):2214-40.

28. Rama-Maceiras $P$, Jokinen J, Kranke P. Stress and burnout in anaesthesia: a real world problem? Curr Opin Anaesthesiol. 2015;28(2):151-8.

29. Roberts DL, Cannon KJ, Wellik KE, Wu Q, Budavari Al. Burnout in inpatientbased versus outpatient-based physicians: a systematic review and metaanalysis. J Hosp Med. 2013;8(11):653-64

30. Toh SG, Ang E, Devi MK. Systematic review on the relationship between the nursing shortage and job satisfaction, stress and burnout levels among nurses in oncology/haematology settings. Int J Evid Based Healthc. 2012; 10(2):126-41.

31. Moher D, Liberati A, Tetzlaff J, Altman DG. Preferred reporting items for systematic reviews and meta-analyses: the PRISMA statement. Int I Surg. 2010;8(5):336-41.

32. Özalp O. Where is the Middle East? The Definition and Classification Problem of the Middle East as a Regional Subsystem in International Relations2011. 5-22 p.

33. Tabatabaei-Malazy O, Shadman Z, Ejtahed HS, Atlasi R, Abdollahi M, Larijan B. Quality of reporting of randomized controlled trials of herbal medicines conducted in metabolic disorders in Middle East countries: Syst Rev. (18736963 (Electronic)).

34. Johnson JM, Carragher R. Interprofessional collaboration and the care and management of type 2 diabetic patients in the Middle East: a systematic review. J Interprof Care. 2018;32(5):621-8.

35. Britannica E. Middle East, The editors of Encyclopedia. Britannica. 2014

36. Dubale BW, Friedman LE, Chemali Z, Denninger JW, Mehta DH, Alem A, et al. Systematic review of burnout among healthcare providers in subSaharan Africa. BMC Public Health. 2019;19(1):1247.
37. Abdulrahman M, Nair SC, Farooq MM, Al Kharmiri A, Al Marzooqi F, Carrick FR. Burnout and depression among medical residents in the United Arab Emirates: a multicenter study. J Family Med Prim Care. 2018;7(2):435-41.

38. Abut YC, Kitapcioglu D, Erkalp K, Toprak N, Boztepe A, Sivrikaya U, et al. Job burnout in 159 anesthesiology trainees. Saudi J Anaesth. 2012;6(1):46-51.

39. Agha A, Mordy A, Anwar E, Saleh N, Rashid I, Saeed M. Burnout among middle-grade doctors of tertiary care hospital in Saudi Arabia. Work (Reading, Mass). 2015;51(4):839-47.

40. Ahmadpanah M, Torabian S, Dastore K, Jahangard L, Haghighi M. Association of occupational burnout and type of personality in Iranian general practitioners. Work (Reading, Mass). 2015;51(2):315-9.

41. Aksoy DY, Durusu Tanriover M, Unal S, Dizdar O, Kalyoncu U, Karakaya J, et al. Burnout syndrome during residency in internal medicine and pediatrics in a country without working time directive. Int J Health Care Qual Assur. 2014:27(3):223-30.

42. Al-Dubai SA, Rampal KG. Prevalence and associated factors of burnout among doctors in Yemen. J Occup Health. 2010:52(1):58-65.

43. Al-Mendalawi MD. Assessment of pediatric residents burnout in a tertiary academic Centre. Saudi medical journal. 2018;39(5):528.

44. Al-Sareai NS, Al-Khaldi YM, Mostafa OA, Abdel-Fattah MM. Magnitude and risk factors for burnout among primary health care physicians in Asir Province, Saudi Arabia. East Mediterr Health J. 2013;19(5):426-34.

45. Al-Youbi RA, Jan MM. Burnout syndrome in pediatric practice. Oman Med J. 2013;28(4):252-4.

46. Aldrees T, Badri M, Islam T, Alqahtani K. Burnout among otolaryngology residents in Saudi Arabia: a multicenter study. J Surg Educ. 2015;72(5):844-8.

47. Aldrees T, Hassouneh B, Alabdulkarim A, Asad L, Alqaryan S, Aljohani E, et al. Burnout among plastic surgery residents. National survey in Saudi Arabia. Saudi Med J. 2017;38(8):832-6.

48. Aldrees TM, Aleissa S, Zamakhshary M, Badri M, Sadat-Ali M. Physician wellbeing: prevalence of burnout and associated risk factors in a tertiary hospital, Riyadh Saudi Arabia. Ann Saudi Med. 2013:33(5):451-6.

49. Alyamani A, Alyamani L, Altheneyan F, Aldhali S, Albaker K, Alshaalan A, et al. Prevalence of burnout among residents at king Abdulaziz Medical City in Riyadh, Saudi Arabia. Int J Med Res Health Sci. 2018;7(12):37-40.

50. Amiri M, Khosravi A, Eghtesadi AR, Sadeghi Z, Abedi G, Ranjbar M, et al. Burnout and its influencing factors among primary health care providers in the north east of Iran. PLoS One. 2016:11(12):e0167648.

51. Arvandi Z, Emami A, Zarghi N, Alavinia SM, Shirazi M, Parikh SV. Linking medical faculty stress/burnout to willingness to implement medical school curriculum change: a preliminary investigation. J Eval Clin Pract. 2016;22(1): 86-92.

52. Ashkar K, Romani M, Musharrafieh U, Chaaya M. Prevalence of burnout syndrome among medical residents: experience of a developing country. Postgrad Med J. 2010;86(1015):266-71.

53. Bar-Sela G, Lulav-Grinwald D, Mitnik I. "Balint group" meetings for oncology residents as a tool to improve therapeutic communication skills and reduce burnout level. J Cancer Educ. 2012;27(4):786-9.

54. Ben-Itzhak S, Dvash J, Maor M, Rosenberg N, Halpern P. Sense of meaning as a predictor of burnout in emergency physicians in Israel: a national survey. Clin Exp Emerg Med. 2015;2(4):217-25.

55. Capraz N, Ozalp DCT, Yalcin M, Can ET, Alkin T. Pressure from superiors and older age increase the risk of burnout syndrome among psychiatric trainees: A nation-wide survey in Turkey. Eur Psychiat. 2017;41:S298-S.

56. Erdur B, Ergin A, Yuksel A, Turkcuer I, Ayrik C, Boz B. Assessment of the relation of violence and burnout among physicians working in the emergency departments in Turkey. Ulus Travma Acil Cerrahi Derg. 2015; 21(3):175-81.

57. Grossman Z, Chodick G, Kushnir T, Cohen HA, Chapnick G, Ashkenazi S. Burnout and intentions to quit the practice among community pediatricians: associations with specific professional activities. Isr J Health Policy Res. 2019;8(1):2.

58. Gül A, Gül H, Özkal UC, Kıncır Z, Gültekin G, Emul HM. The relationship between sluggish cognitive tempo and burnout symptoms in psychiatrists with different therapeutic approaches. Psychiatry Res. 2017;252:284-8.

59. Haik J, Brown S, Liran A, Visentin D, Sokolov A, Zilinsky I, et al. Burnout and compassion fatigue: prevalence and associations among Israeli burn clinicians. Neuropsychiatr Dis Treat. 2017;13:1533-40.

60. Hameed TK, Masuadi E, Al Asmary NA, Al-Anzi FG, Al Dubayee MS. A study of resident duty hours and burnout in a sample of Saudi residents. BMC Med Educ. 2018;18(1):180. 
61. Hasan HI, Nooh Y, Alsayyad AS. Prevalence and factors affecting burnout among secondary care doctors in Bahrain- a cross sectional study. Int J Med Res Health Sci. 2015;4(2):401-6.

62. Jalili M, Roodsari GS, Nia AB. Burnout and associated factors among Iranian emergency medicine practitioners. Iran J Public Health. 2013;42(9):1034-42.

63. Jamjoom RS, Park YS. Assessment of pediatric residents burnout in a tertiary academic Centre. Saudi Med J. 2018;39(3):296-300.

64. Karaoglu N, Pekcan S, Durduran Y, Mergen H, Odabasi D, Ors R. A sample of paediatric residents' loneliness-anxiety-depression-burnout and job satisfaction with probable affecting factors. J Pak Med Assoc. 2015;65(2): 183-91.

65. Keinan G, Melamed S. Personality characteristics and proneness to burnout: a study among internists. Stress Med. 1987;3(4):307-15.

66. Kosan Z, Calikoglu EO, Guraksin A. Levels of burnout and their associated factors among physicians working in Northeast Anatolia. Niger J Clin Pract. 2018;21(7):875-81

67. Kotb AA, Mohamed KA, Kamel MH, Ismail MA, Abdulmajeed AA Comparison of burnout pattern between hospital physicians and family physicians working in Suez Canal University Hospitals. Pan Afr Med J. 2014 18:164.

68. Kotb AA, Mohamed KA-E, Kamel MH, Ismail MAR, Abdulmajeed AA. Evaluating the effect of an educational program on level of professional burnout among family physicians in the Faculty of Medicine-Suez Canal University. Arab J Psych. 2014;25(1):52-60.

69. Kushnir $\mathrm{T}$, Cohen $\mathrm{AH}$. Job structure and burnout among primary care pediatricians. Work (Reading, Mass). 2006;27(1):67-74.

70. Pirincci E, Vicdanli SS. Burnout levels of assistant physicians working at a TURKISH UNIVERSITY hospital. Southeast Asian J Trop Med Public Health. 2015;46(2):330-8.

71. Sadat-Ali M, AI-Habdan IM, Al-Dakheel DA, Shriyan D. Are orthopedic surgeons prone to burnout? Saudi Med J. 2005;26(8):1180-2.

72. Salem M, Taher M, Alsaadi H, Alnema A, Al-Abdulla S. Prevalence and determinants of burnout among primary healthcare physicians in Qatar. World Fam Med. 2018;16(7):22-8.

73. Salem R, Akel R, Fakhri G, Tfayli A. Burnout among Lebanese oncologists: prevalence and risk factors. Asian Pac J Cancer Prev. 2018:19(8):2135-9.

74. Shams T, El-Masry R. Job stress and burnout among academic career Anaesthesiologists at an Egyptian University hospital. Sultan Qaboos Univ Med J. 2013;13(2):287-95.

75. Shinan-Altman S, Cohen M, Rasmussen V, Turnell A, Butow P. Burnout among psychosocial oncologists in Israel: the direct and indirect effects of job demands and job resources. Palliat Support Care. 2018;16(6):677-84.

76. Soltanifar A, Pishbin E, Attaran Mashhadi N, Najaf Najafi M, Siahtir M. Burnout among female emergency medicine physicians: a nationwide study. Emerg Med Australas. 2018;30(4):517-22.

77. Tarcan GY, Tarcan M, Top M. An analysis of relationship between burnout and job satisfaction among emergency health professionals. Total Qual Manag Bus Excell. 2017;28(11-12):1339-56.

78. Taycan O, Taycan SE, Celik C. Relationship of burnout with personality, alexithymia, and coping behaviors among physicians in a semiurban and rural area in Turkey. Arch Environ Occup Health. 2014;69(3):159-66.

79. Turgut N, Karacalar S, Polat C, Kiran O, Gultop F, Kalyon ST, et al. Burnout syndrome during residency. Turk J Anaesthesiol Reanim. 2016;44(5):258-64.

80. Tzischinsky O, Zohar D, Epstein R, Chillag N, Lavie P. Daily and yearly burnout symptoms in Israeli shift work residents. J Hum Ergol. 2001;30(1-2): 357-62

81. Afana A, Ghannam J, Ho EY, Al-Khal A, Al-Arab B, Bylund CL. Burnout and sources of stress among medical residents at Hamad Medical Corporation, Qatar. East Mediterr Health J. 2017;23(1):40-5.

82. Bawakid K, Abdulrashid O, Mandoura N, Shah HB, Ibrahim A, Akkad NM, et al. Burnout of Physicians Working in Primary Health Care Centers under Ministry of Health Jeddah, Saudi Arabia. Cureus. 2017;9(11).

83. Ghannam J, Afana A, Ho EY, Al-Khal A, Bylund CL. The impact of a stress management intervention on medical residents' stress and burnout. Int J Stress Manage. 2019

84. Al-Shuhail AS, Al-Saleh S, Al-saleh SS, Elhassan I, Wajid S. Prevalence of burnout among National Guard Health Affairs Physicians in Dammam, Saudi Arabia - a cross-sectional study. Asian J Pharm. 2017;11(4):S924-S9.

85. Haber Y, Palgi Y, Hamama-Raz Y, Shrira A, Ben-Ezra M. Predictors of professional quality of life among physicians in a conflict setting: the role of risk and protective factors. Isr J Psychiatry Relat Sci. 2013;50(3):174-80.
86. Talih F, Warakian R, Ajaltouni J, Shehab AA, Tamim H. Correlates of depression and burnout among residents in a Lebanese Academic Medical Center: a cross-sectional study. Acad Psychiatry. 2016;40(1):38-45.

87. Kushnir T, Greenberg D, Madjar N, Hadari I, Yermiahu Y, Bachner YG. Is burnout associated with referral rates among primary care physicians in community clinics? Fam Pract. 2014;31(1):44-50.

88. Kushnir T, Cohen AH. Positive and negative work characteristics associated with burnout among primary care pediatricians. Pediatr Int. 2008;50(4):546-51.

89. Granek L, Krzyzanowska MK, Nakash O, Cohen M, Ariad S, Barbera L, et al. Gender differences in the effect of grief reactions and burnout on emotional distress among clinical oncologists. Cancer. 2016;122(23):3705-14.

90. Granek L, Ben-David M, Nakash O, Cohen M, Barbera L, Ariad S, et al. Oncologists' negative attitudes towards expressing emotion over patient death and burnout. Support Care Cancer. 2017;25(5):1607-14.

91. Abushaikha L, Saca-Hazboun H. Job satisfaction and burnout among Palestinian nurses. East Mediterr Health J. 2009, 15;(1):190-7.

92. Ahmadi O, Azizkhani R, Basravi M. Correlation between workplace and occupational burnout syndrome in nurses. Adv Biomed Res. 2014;3:44.

93. Akkuş Y, Karacan Y, Göker H, Aksu S. Determination of burnout levels of nurses working in stem cell transplantation units in Turkey. Nurs Health Sci. 2010;12(4):444-9.

94. Akman O, Ozturk C, Bektas M, Ayar D, Armstrong MA. Job satisfaction and burnout among paediatric nurses. J Nurs Manag. 2016;24(7):923-33.

95. Al-Turki HA. Saudi Arabian nurses. Are they prone to burnout syndrome? Saudi Med J. 2010;31(3):313-6.

96. Al-Turki HA, Al-Turki RA, Al-Dardas HA, Al-Gazal MR, Al-Maghrabi GH, Al-Enizi $\mathrm{NH}$, et al. Burnout syndrome among multinational nurses working in Saudi Arabia. Ann Afr Med. 2010;9(4):226-9.

97. Alharbi J, Wilson R, Woods C, Usher K. The factors influencing burnout and job satisfaction among critical care nurses: a study of Saudi critical care nurses. J Nurs Manag. 2016;24(6):708-17.

98. Alimoglu MK, Donmez L. Daylight exposure and the other predictors of burnout among nurses in a university hospital. Int J Nurs Stud. 2005;42(5):549-55.

99. Altun I. Burnout and nurses' personal and professional values. Nurs Ethics. 2002;9(3):269-78.

100. Anwar MM, Elareed HR. Burnout among Egyptian nurses. J Public Health. 2017;25(6):693-7.

101. Arslan Yurumezoglu H, Kocaman G. Predictors of nurses' intentions to leave the organisation and the profession in Turkey. J Nurs Manag. 2016;24(2):235-43.

102. Azmoon $\mathrm{H}$, Nodooshan $\mathrm{HS}$, Jalilian $\mathrm{H}$, Choobineh A, Shouroki FK. The Relationship Between Fatigue and Job Burnout Dimensions in Hospital Nurses. Health Scope. 2018;7(2).

103. Bagheri Hosseinabadi M, Ebrahimi MH, Khanjani N, Biganeh J, Mohammadi S, Abdolahfard M. The effects of amplitude and stability of circadian rhythm and occupational stress on burnout syndrome and job dissatisfaction among irregular shift working nurses. J Clin Nurs. 2019.

104. Bakir B, Ozer M, Ozcan CT, Cetin M, Fedai T. The association between burnout, and depressive symptoms in a Turkish military nurse sample. Klinik Psikofarmakoloji Bülteni / Bulletin of Clinical Psychopharmacology. 2010; 20(2):160-3

105. Chayu T, Kreitler S. Burnout in nephrology nurses in Israel. Nephrol Nurs J. 2011;38(1):65-77 quiz 8.

106. Darban F, Balouchi A, Narouipour A, Safarzaei E, Shahdadi H. Effect of Communication Skills Training on the Burnout of Nurses: A Cross-Sectional Study. J Clin Diagn Res. 2016;10(4):Ic01-ic4.

107. Demir A, Ulusoy M, Ulusoy MF. Investigation of factors influencing burnout levels in the professional and private lives of nurses. Int J Nurs Stud. 2003: 40(8):807-27.

108. Dor A, Mashiach Eizenberg M, Halperin O. Hospital nurses in comparison to community nurses: motivation, empathy, and the mediating role of burnout. Can J Nurs Res. 2018:844562118809262.

109. Emold C, Schneider N, Meller I, Yagil Y. Communication skills, working environment and burnout among oncology nurses. Eur J Oncol Nurs. 2011; 15(4):358-63.

110. Farahbod F, Goudarzvand Chegini M, Kouchakinejad Eramsadati L, Mohtasham-Amiri Z. The association between social capital and burnout in nurses of a trauma referral teaching hospital. Acta Med Iran. 2015;53(4):214-9.

111. Gholami T, Pahlavian AH, Akbarzadeh M, Motamedzade M, Moghaddam RH. The role of burnout syndrome as a mediator for the effect of psychosocial risk factors on the intensity of musculoskeletal disorders: a structural equation modeling approach. Int J Occup Saf Ergon. 2016;22(2):283-90. 
112. Gunusen NP, Ustun B. An RCT of coping and support groups to reduce burnout among nurses. Int Nurs Rev. 2010;57(4):485-92.

113. Hamaideh SH. Burnout, social support, and job satisfaction among Jordanian mental health nurses. Issues Ment Health Nurs. 2011;32(4):234-42.

114. lecovich E, Avivi M. Agism and burnout among nurses in long-term care facilities in Israel. Aging Ment Health. 2017;21(3):327-35.

115. Ihan MN, Durukan E, Taner E, Maral I, Bumin MA. Burnout and its correlates among nursing staff: questionnaire survey. J Adv Nurs. 2008;61(1):100-6.

116. Kapucu SS, Akkus Y, Akdemir N, Karacan Y. The burnout and exhaustion levels of nurses working in haemodialysis units. J Ren Care. 2009;35(3):134-40.

117. Karadag Arli S, Bakan AB, Erisik E. An investigation of the relationship between Nurses' views on spirituality and spiritual care and their level of burnout. J Holist Nurs. 2017:35(3):214-20.

118. Karakoc A, Yilmaz M, Alcalar N, Esen B, Kayabasi H, Sit D. Burnout syndrome among hemodialysis and peritoneal Dialysis nurses. Iran J Kidney Dis. 2016; 10(6):395-404.

119. Karaman Ozlu Z, Cay Yayla A, Gumus K, Khaghanyrad E. Comparison of nurses in two different cultures: who experiences more burnout. J Perianesth Nurs. 2017;32(3):238-44.

120. Kavurmaci M, Cantekin I, Tan M. Burnout levels of hemodialysis nurses. Ren Fail. 2014;36(7):1038-42.

121. Kutluturkan S, Sozeri E, Uysal N, Bay F. Resilience and burnout status among nurses working in oncology. Ann General Psychiatry. 2016;15:33.

122. Kızılcı S, Erdoğan V, Sözen E. The influence of selected personality and workplace features on burnout among nurse academics. TOJET: The Turkish Online Journal of Educational Technology. 2012;11(4):307-14.

123. Moghaddasi J, Mehralian H, Aslani Y, Masoodi R, Amiri M. Burnout among nurses working in medical and educational centers in Shahrekord, Iran. Iran J Nurs Midwifery Res. 2013;18(4):294-7.

124. Mohammadpoorasl A, Maleki A, Sahebihagh MH. Prevalence of professional burnout and its related factors among nurses in Tabriz in 2010. Iran J Nurs Midwifery Res. 2012;17(7):524-9.

125. Mudallal RH, Othman WM, Al Hassan NF. Nurses' Burnout: The Influence of Leader Empowering Behaviors, Work Conditions, and Demographic Traits. Inquiry. 2017:54:46958017724944.

126. Nayeri ND, Negarandeh R, Vaismoradi M, Ahmadi F, Faghihzadeh S. Burnout and productivity among Iranian nurses. Nurs Health Sci. 2009; 11(3):263-70

127. Ozbas AA, Tel $H$. The effect of a psychological empowerment program based on psychodrama on empowerment perception and burnout levels in oncology nurses: psychological empowerment in oncology nurses. Palliat Support Care. 2016;14(4):393-401.

128. Ozden D, Karagozoglu S, Yildirim G. Intensive care nurses' perception of futility: job satisfaction and burnout dimensions. Nurs Ethics. 2013;20(4):436-47.

129. Ozgur G, Tektas P. An examination of the correlation between nurses' organizational trust and burnout levels. Appl Nurs Res. 2018;43:93-7.

130. Palazoglu CA, Koc Z. Ethical sensitivity, burnout, and job satisfaction in emergency nurses. Nurs Ethics. 2017;969733017720846.

131. Rezaei O, Habibi K, Arab Ghahestany D, Sayadnasiri M, Armoon B, Khan V, et al. Factors related to job burnout among nurses in the Razi psychiatric hospital, Iran. Int J Adolesc Med Health. 2018.

132. Ron $\mathrm{P}$, Shamai $\mathrm{M}$. The impact of ongoing national terror on the community of hospital nurses in Israel. Community Ment Health J. 2014:50(3):354-61.

133. Sabancıogullari S, Dogan S. Effects of the professional identity development programme on the professional identity, job satisfaction and burnout levels of nurses: a pilot study. Int J Nurs Pract. 2015;21(6):847-57.

134. Sahraian A, Fazelzadeh A, Mehdizadeh AR, Toobaee SH. Burnout in hospital nurses: a comparison of internal, surgery, psychiatry and burns wards. Int Nurs Rev. 2008;55(1):62-7.

135. Shahriari M, Shamali M, Yazdannik A. The relationship between fixed and rotating shifts with job burnout in nurses working in critical care areas. Iran J Nurs Midwifery Res. 2014;19(4):360-5.

136. Shamali M, Shahriari M, Babaii A, Abbasinia M. Comparative study of job burnout among critical care nurses with fixed and rotating shift schedules. Nurs Midwifery Stud. 2015;4(3):e27766.

137. Sorour AS, El-Maksoud MM. Relationship between musculoskeletal disorders, job demands, and burnout among emergency nurses. Adv Emerg Nurs J. 2012;34(3):272-82.

138. Soroush F, Zargham-Boroujeni A, Namnabati M. The relationship between nurses' clinical competence and burnout in neonatal intensive care units. Iran J Nurs Midwifery Res. 2016;21(4):424-9.
139. Taleghani F, Ashouri E, Saburi M. Empathy, burnout, demographic variables and their relationships in oncology nurses. Iran J Nurs Midwifery Res. 2017; 22(1):41-5.

140. Tekindal B, Tekindal MA, Pinar G, Ozturk F, Alan S. Nurses' burnout and unmet nursing care needs of patients' relatives in a Turkish state hospital. Int J Nurs Pract. 2012;18(1):68-76.

141. Topbas E, Bay H, Turan BB, Citlak U, Emir AH, Erdogan TK, et al. The effect of perceived organisational justice on job satisfaction and burnout levels of haemodialysis nurses. J Ren Care. 2019.

142. Tuna R, Baykal U. The relationship between job stress and burnout levels of oncology nurses. Asia Pac J Oncol Nurs. 2014;1(1):33-9.

143. Yousefy AR, Ghassemi GR. Job burnout in psychiatric and medical nurses in Isfahan, Islamic Republic of Iran. East Mediterr Health J. 2006; 12(5):662-9.

144. Abarghouei MR, Sorbi MH, Abarghouei M, Bidaki R, Yazdanpoor S. A study of job stress and burnout and related factors in the hospital personnel of Iran. Electron Physician. 2016;8(7):2625-32.

145. Abdo SA, El-Sallamy RM, El-Sherbiny AA, Kabbash IA. Burnout among physicians and nursing staff working in the emergency hospital of Tanta University, Egypt. East Mediterr Health J. 2016;21(12):906-15.

146. Alacacioglu A, Yavuzsen T, Dirioz M. Oztop I, Yilmaz U. burnout in nurses and physicians working at an oncology department. Psycho Oncol. 2009; 18(5):543-8.

147. Alameddine M, Kazzi A, El-Jardali F, Dimassi H, Maalouf S. Occupational violence at Lebanese emergency departments: prevalence, characteristics and associated factors. J Occup Health. 2011;53(6):455-64.

148. Alameddine M, Saleh S, El-Jardali F, Dimassi H, Mourad Y. The retention of health human resources in primary healthcare centers in Lebanon: a national survey. BMC Health Serv Res. 2012;12:419.

149. Alameddine M, Baroud M, Kharroubi S, Hamadeh R, Ammar W, Shoaib H, et al. Investigating the job satisfaction of healthcare providers at primary healthcare centres in Lebanon: a national cross-sectional study. Health Soc Care Comm. 2017;25(6):1805-16.

150. Ashtari Z, Farhady Y, Khodaee MR. Relationship between job burnout and work performance in a sample of Iranian mental health staff. Afr J Psychiatry. 2009;12(1):71-4.

151. Bijari B, Abassi A. Prevalence of burnout syndrome and associated factors among rural health workers (Behvarzes) in South Khorasan. Iran Red Crescent Med J. 2016;18(10):e25390.

152. Calgan Z, Aslan D, Yegenoglu S. Community pharmacists' burnout levels and related factors: an example from Turkey. Int J Clin Pharm. 2011;33(1):92-100.

153. Demirci S, Yildirim YK, Ozsaran Z, Uslu R, Yalman D, Aras AB. Evaluation of burnout syndrome in oncology employees. Med Oncol. 2010;27(3):968-74.

154. Devebakan N, Dogan O, Ceylan V, Akin E, Kose S. Relationship between temperament and character dimensions of personality and burnout and management in healthcare organization workers. Psychiatry Clin Psychopharmacol. 2018;28(1):73-9.

155. Gökçen C, Zengin S, Oktay MM, Alpak G, Al B, Yıldırım C. Burnout, job satisfaction and depression in the healthcare personnel who work in the emergency department. Anadolu Psikiyatri Dergisi. 2013;14(2):122-8.

156. Gulalp B, Karcioglu O, Sari A, Koseoglu Z. Burnout: need help? J Occup Med Toxicol. 2008:3:32

157. Guveli H, Anuk D, Oflaz S, Guveli ME, Yildirim NK, Ozkan M, et al. Oncology staff: burnout, job satisfaction and coping with stress. Psycho Oncol. 2015; 24(8):926-31.

158. Hamdan M, Hamra AA. Burnout among workers in emergency Departments in Palestinian hospitals: prevalence and associated factors. BMC Health Serv Res. 2017;17(1):407.

159. Hosseiniarzfuni SM, Gorji AMH, Ranjabar M, Giorji RH, Rostamnejad M. Job burnout among technicians and nurses of northern Iran. Eur J Ther. 2015; 21(2):114-7

160. Kabir MJ, Heidari A, Etemad K, Gashti AB, Jafari N, Honarvar MR, et al. Job burnout, job satisfaction, and related factors among health Care Workers in Golestan Province Iran. Electron Physician. 2016;8(9):2924-30.

161. Komur I, Ozdemirel RO, Ozver I, Baspinar B, Demir M, Gonen F, et al. Posttraumatic stress and burnout symptoms in forensic doctors and staff in a mortuary. Am J Forensic Med Pathol. 2017;38(3):184-8.

162. Malakouti SK, Nojomi M, Salehi M, Bijari B. Job stress and burnout syndrome in a sample of rural health workers, behvarzes, in Tehran, Iran. Iran J Psychiatry. 2011;6(2):70-4. 
163. Tarcan M, Hikmet N, Schooley B, Top M, Tarcan GY. An analysis of the relationship between burnout, socio-demographic and workplace factors and job satisfaction among emergency department health professionals. Appl Nurs Res. 2017;34:40-7.

164. Tekin A, Karadag H, Yayla S. The relationship between burnout symptoms and type D personality among health care professionals in Turkey. Arch Environ Occup Health. 2017;72(3):173-7.

165. Tunc T, Kutanis RO. Role conflict, role ambiguity, and burnout in nurses and physicians at a university hospital in Turkey. Nurs Health Sci. 2009;1 1(4):410-6.

166. Al-Alawi M, Al-Sinawi H, Al-Qubtan A, Al-Lawati J, Al-Habsi A, Al-Shuraiqi M, et al. Prevalence and determinants of burnout syndrome and depression among medical students at Sultan Qaboos University: a cross-sectional analytical study from Oman. Arch Environ Occup Health. 2017:1-10.

167. Almalki SA, Almojali Al, Alothman AS, Masuadi EM, Alaqeel MK. Burnout and its association with extracurricular activities among medical students in Saudi Arabia. Int J Med Educ. 2017;8:144-50.

168. Altannir Y, Alnajjar W, Ahmad SO, Altannir M, Yousuf F, Obeidat A, et al. Assessment of burnout in medical undergraduate students in Riyadh, Saudi Arabia. BMC Med Educ. 2019;19(1):34.

169. Ebrahimi S, Atazadeh F. Medical Students' occupational burnout and its relationship with professionalism. J Adv Med Educ Prof. 2018;6(4):162-7.

170. Fares J, Saadeddin Z, Al Tabosh H, Aridi H, El Mouhayyar C, Koleilat MK, et al. Extracurricular activities associated with stress and burnout in preclinical medical students. J Epidemiol Glob Health. 2016;6(3):177-85.

171. Talih F, Daher M, Daou D, Ajaltouni J. Examining burnout, depression, and attitudes regarding drug use among Lebanese medical students during the 4 years of medical school. Acad Psychiatry. 2018;42(2):288-96.

172. Sevencan F, Cayir E, Uner S. Burnout status of interns and associated factors. Cah Sociol Demogr Med. 2010;50(4):501-15.

173. Fares J, Al Tabosh H, Saadeddin Z, El Mouhayyar C, Aridi H. Stress, burnout and coping strategies in preclinical medical students. N Am J Med Sci. 2016; 8(2):75-81.

174. Kristensen TS, Borritz M, Villadsen E, Christensen KB. The Copenhagen burnout inventory: a new tool for the assessment of burnout. Work Stress. 2005;19(3):192-207.

\section{Publisher's Note}

Springer Nature remains neutral with regard to jurisdictional claims in published maps and institutional affiliations.

Ready to submit your research? Choose BMC and benefit from:

- fast, convenient online submission

- thorough peer review by experienced researchers in your field

- rapid publication on acceptance

- support for research data, including large and complex data types

- gold Open Access which fosters wider collaboration and increased citations

- maximum visibility for your research: over $100 \mathrm{M}$ website views per year

At $\mathrm{BMC}$, research is always in progress.

Learn more biomedcentral.com/submissions 